\title{
23. Freiwilliges Engagement und informelle Unterstützungsleistungen von Personen mit Migrationshintergrund
}

\author{
Claudia Vogel, Julia Simonson \& Clemens Tesch-Römer
}

\section{Kernaussagen}

Die Anteile freiwillig Engagierter sind bei Menschen mit und ohne Migrationshintergrund unterschiedlich. Allerdings ist bei Menschen mit Migrationshintergrund, die in Deutschland geboren sind und die deutsche Staatsangehörigkeit besitzen, der Anteil der Engagierten mit 43,2 Prozent ähnlich hoch wie bei jenen ohne Migrationshintergrund (46,8 Prozent). Dagegen ist der Anteil der Engagierten unter den Menschen mit Migrationshintergrund, die zwar in Deutschland geboren sind, aber nicht die deutsche Staatsangehörigkeit haben, deutlich geringer (31,1 Prozent). Am geringsten ist der Anteil der Engagierten bei Personen mit eigener Migrationserfahrung (mit deutscher Staatsangehörigkeit: 26,4 Prozent; ohne deutsche Staatsangehörigkeit: 21,7 Prozent).

Unterschiede im Anteil Engagierter zwischen Menschen mit und ohne Migrationshintergrund sind nur zu geringen Teilen auf Unterschiede hinsichtlich Alter, Bildung und Einkommen zurückzuführen. Vergleicht man die Menschen mit Migrationshintergrund mit jenen ohne Migrationshintergrund und berücksichtigt dabei Unterschiede in der Lebenssituation (zum Beispiel Alter, Bildung, Einkommen), so bleiben die gefundenen Gruppenunterschiede bezüglich des freiwilligen Engagements stabil. Offensichtlich sind prägende Erfahrungen in Kindheit und Jugend, die sich für Personen mit und ohne eigene Migrationserfahrung unterscheiden, von erheblicher Bedeutung für das freiwillige Engagement. Auch die Staatsangehörigkeit ist bedeutsam. Beide Merkmale tragen zur Vorhersage des Engagements bei, auch wenn Unterschiede in der sozioökonomischen Situation berücksichtigt werden.

Die Engagementbereitschaft nicht-engagierter Personen ist bei Menschen mit Migrationshintergrund größer als bei Deutschen ohne Migrationshintergrund. 13,6 Prozent der Menschen mit Migrationshintergrund sind sicher bereit, sich künftig engagieren zu wollen, im Vergleich zu 10,8 Prozent ohne Migrationshintergrund. Menschen mit Migrationshintergrund sind darüber hinaus wesentlich häufiger bereit, sich künftig vielleicht zu engagieren (54,5 Prozent zu 45,0 Prozent bei Personen ohne Migrationshintergrund).

Die Unterschiede zwischen verschiedenen Bevölkerungsgruppen nach Art des Migrationshintergrundes sind bezüglich der informellen Unterstützungsleistungen geringer als bezüglich des freiwilligen Engagements. Menschen mit Migrationshintergrund leisten im Vergleich zu Menschen ohne Migrationshintergrund zu etwas höheren Anteilen Kinderbetreuung nicht-eigener Kinder, zu etwas geringeren Anteilen instrumentelle Hilfen und zu etwa gleichen Anteilen Pflege- und Betreuungsleistungen im außerfamilialen sozialen Nahraum. 


\subsection{Einleitung}

Das freiwillige Engagement und die öffentliche Aktivität von Menschen mit Migrationshintergrund können als Indikatoren für die gesellschaftlichen Teilhabechancen in der bundesdeutschen Gesellschaft verstanden werden. Freiwilliges Engagement hat für Menschen, die aus ihrem Herkunftsland in eine neue Heimat eingewandert sind, eine doppelte Bedeutung (Bundesamt für Migration und Flüchtlinge (BAMF) 2010: 161): Zum einen übernehmen engagierte Migrantinnen und Migranten wichtige gesellschaftliche Aufgaben (Engagement kann also als Zeichen von Integration verstanden werden), zum anderen ist freiwilliges Engagement auch ein Weg zu Teilhabe und Mitgestaltung der Gesellschaft (kann also einen Weg in die Integration bilden). Sind die Anteile freiwillig Engagierter, die gewählten Engagementbereiche und die Ausgestaltung des Engagements bei Menschen mit und ohne Migrationshintergrund ähnlich, kann dies ebenso mit Blick auf die Integrationsleistung der Migrantinnen und Migranten interpretiert werden als auch mit Blick auf die Integrationsleistung der Gesellschaft der Bundesrepublik Deutschland. Beide Perspektiven werden in der Engagementforschung thematisiert, in der Regel als Engagement von Migrantinnen und Migranten beziehungsweise als Engagement für Migrantinnen und Migranten (Huth 2011). Unterschiede können hingegen auf Handlungsbedarfe verweisen, auch seitens der zivilgesellschaftlichen Organisationen und der Politik.

Dabei gibt es gute Gründe, Unterschiede bezüglich der gesellschaftlichen Teilhabe von Menschen mit und ohne Migrationshintergrund zu erwarten. Wer nach Deutschland einwandert und hier ein neues Leben beginnt, sich also in einer möglicherweise fremden Sprache und unter Beachtung der Regeln einer anderen Kultur zurecht finden muss, hat viele Aufgaben zu bewältigen. Freiwilliges Engagement gehört dabei zu den Aktivitäten, die meist erst im Laufe der Zeit in ihrer Bedeutung kennengelernt, erfahren und ausgeübt werden, einmal abgesehen von Beispielen aus der aktuellen Flüchtlingsarbeit, bei der auch Flüchtlinge selbst als ehrenamtliche Dolmetscherinnen und Dolmetscher oder Helferinnen und Helfer etwa in der medizinischen Versorgung anderer Flüchtlinge tätig werden. Angesichts der großen Unterschiedlichkeit der Menschen mit Migrationshintergrund sollte man differenzierte Analysen vornehmen: Sie kommen aus verschiedenen Herkunftsländern oder -kulturen, sprechen unterschiedliche Sprachen, sind in unterschiedlichen Lebensphasen nach Deutschland gekommen (oder in Deutschland geboren worden) und leben unter unterschiedlichen rechtlichen Bedingungen in Deutschland (Bundesamt für Migration und Flüchtlinge (BAMF) 2016). Möglicherweise sind die Unterschiede innerhalb der Gruppe von Menschen mit Migrationshintergrund größer als die Unterschiede zwischen Menschen mit Migrationshintergrund und Menschen ohne Migrationshintergrund.

Gruppen von Menschen mit Migrationshintergrund: Wir unterscheiden vier Gruppen von Menschen mit Migrationshintergrund. Zur Bildung dieser Gruppen haben wir aus der Vielfalt möglicher Beschreibungsdimensionen zwei ausgewählt, nämlich die Zuwanderungserfahrung und die Staatsbürgerschaft (entsprechend dem Migrationshintergrund-Konzept des Statistischen Bundesamtes, Statistisches Bundesamt 2013: 5). Andere wichtige Dimensionen der Migration berücksichtigen wir im vorliegenden Kapitel nur am Rande. Dazu gehören Sprache, Kultur oder Religionszugehörigkeit. Trotz unseres Wissens um die Gefahr der Stereotypisierung: Auch wir (müssen) vereinfachen, denn die Komplexität des Phänomens ,Migration' ist zu groß, um alle wichtigen Dimensionen zugleich zu erfassen.

Eine erste Dimension ist die Zuwanderungserfahrung, also die Frage, ob eine Person außerhalb Deutschlands oder in Deutschland geboren ist. Eine zweite Dimension ist die Staatsangehörigkeit: Während eine Gruppe von Migrantinnen und Migranten (noch) keine deutsche Staats- 
angehörigkeit hat, verfügt eine zweite Gruppe (bereits) darüber. Kombiniert man diese beiden Dimensionen, so erhält man vier Gruppen:
a) Menschen mit eigener Migrationserfahrung ohne deutsche Staatsangehörigkeit
b) Menschen mit eigener Migrationserfahrung mit deutscher Staatsangehörigkeit
c) Menschen mit Migrationshintergrund, die in Deutschland geboren sind aber keine deut- sche Staatsangehörigkeit haben
d) Menschen mit Migrationshintergrund, die in Deutschland geboren sind und die deutsche Staatsangehörigkeit haben

Die ,Deutschen ohne Migrationshintergrund dienen in den Analysen dieses Kapitels als Vergleichsgruppe. Insgesamt leben in Deutschland (bei einer Gesamtbevölkerung von 80,9 Millionen im Jahr 2014) rund 16,4 Millionen Menschen mit Migrationshintergrund. Mehr als jede fünfte in Deutschland lebende Person hat einen Migrationshintergrund. Laut Statistischem Bundesamt (2015) haben davon etwa 10,9 Millionen eine eigene Zuwanderungserfahrung (diese Gruppe wird auch als Migrantinnen und Migranten bezeichnet), 5,5 Millionen sind in Deutschland geboren worden. Etwa 7,2 Millionen Menschen mit Migrationshintergrund haben ausschließlich eine ausländische Staatsangehörigkeit, etwa 9,2 Millionen haben (auch) die deutsche Staatsbürgerschaft. ${ }^{1}$

Was bedeutet es nun, die Migrationserfahrung selbst gemacht zu haben - oder aber als Kind einer Familie mit Migrationserfahrung in Deutschland aufgewachsen zu sein? Menschen, die selbst die Erfahrung des Auswanderns aus dem Herkunftsland (und des Einwanderns nach Deutschland) gemacht haben, sind in der Regel in ihrem Herkunftsland aufgewachsen, sind dort zur Schule gegangen, sprechen dessen Sprache und sind mit einer Gesellschaft vertraut, deren

1 Mehr als 1,5 Millionen Menschen in Deutschland haben neben der deutschen auch noch eine andere Staatsangehörigkeit (Statistisches Bundesamt 2015). soziale, politische, wirtschaftliche und religiöse Systeme sich von denen in Deutschland zum Teil stark unterscheiden. Menschen mit eigener Migrationserfahrung sind allen Chancen und Belastungen des Wechselns zwischen den Welten direkt ausgesetzt. In einer solchen Lebenssituation sind die Existenzsicherung durch einen Beruf und die Sorge um die eigene Familie möglicherweise von höherer Bedeutung als die Übernahme eines freiwilligen Engagements. Wer im Gegensatz hierzu in Deutschland als Kind von Eltern mit eigener Migrationserfahrung geboren wurde, wächst nicht allein in der Kultur der Eltern auf, sondern auch in die deutsche Gesellschaft hinein. Neben Schule und Berufsausbildung bieten Sport und Freizeit vielfältige Möglichkeiten, sich freiwillig zu betätigen. Möglicherweise verstärkt sich über die Generationen die Integrationsleistung der deutschen Gesellschaft: Während Migrantinnen und Migranten der, ersten Generation' (also Personen mit Zuwanderungserfahrung) sich in geringerem Maß freiwillig engagieren, sind Menschen mit Migrationshintergrund der, zweiten Generation' (also Personen, die in Familien mit Migrationserfahrung in Deutschland geboren wurden) zu höheren Anteilen freiwillig engagiert.

Die deutsche Staatsbürgerschaft ist ein zentrales Bestimmungsstück der Integration. Als Staatsbürgerin oder Staatsbürger ist eine Person berechtigt, über die Zukunft des Landes mitzubestimmen: Sie ist als Wählerin oder Wähler Souverän des Landes. Für bestimmte freiwillige oder ehrenamtliche Aufgaben wird die deutsche Staatsangehörigkeit vorausgesetzt. Mit Blick auf freiwilliges Engagement hat die Staatsbürgerschaft auf den ersten Blick also die Funktion einer ,Zugangsberechtigung. Beispielsweise sind das Schöffenamt und die Ratsmitgliedschaft in einem Gemeinderat an die deutsche Staatsangehörigkeit geknüpft. Die fehlende deutsche Staatsangehörigkeit stellt eine Zugangsbarriere zum Engagement dar, wenn dieser Zugang formal an sie geknüpft ist. Für Menschen mit Migrationshintergrund ohne deutsche Staatsbürgerschaft (Ausländerinnen und Ausländern) ist somit eine geringere Beteiligung in diesen Formen 
des Engagements zu erwarten. Aber die Staatsangehörigkeit geht weit über die Bedeutung der Zugangsvoraussetzung zu bestimmten Ämtern hinaus: Sie ist das sichtbare Zeichen der Zugehörigkeit zu einem Staat. Es ist anzunehmen, dass wer die Staatsbürgerschaft des Landes besitzt, in dem sie oder er lebt, sich dem Land und der Gesellschaft stärker verbunden fühlt, die Absicht hat, hier zu bleiben und daher möglicherweise auch eher bereit ist, sich freiwillig zu engagieren und die Gesellschaft mitzugestalten. Befunde aus dem Freiwilligenmonitor der Schweiz stützen diese These, dort engagieren sich die Eingebürgerten zu höheren Anteilen als die Ausländerinnen und Ausländer, aber zu geringeren Teilen als die gebürtigen Schweizerinnen und Schweizer (Freitag, Manatschal, Ackermann \& Ackermann 2016).

Der Begriff des bürgerschaftlichen Engagements, der häufig austauschbar zum freiwilligen Engagement verwendet wird, benennt diesen Aspekt direkt: Die bundesdeutsche Zivilgesellschaft wird von den bundesdeutschen Staatsbürgerinnen und Staatsbürgern geprägt. (Spät-)Aussiedlerinnen und (Spät-)Aussiedler ${ }^{2}$, die bei der Übersiedlung aus einem der Länder der ehemaligen Sowjetunion nach Deutschland die deutsche Staatsbürgerschaft erhalten, sind möglicherweise - trotz eigener Migrationserfahrung - eher bereit sich freiwillig zu engagieren als Menschen mit eigener Migrationserfahrung, die die deutsche Staatsbürgerschaft nicht besitzen. Entsprechend kann man auch vermuten, dass das Aufwachsen in Deutschland sich als besonders teilhabefördernd erweist, wenn ein Kind, das in einer Familie mit Migrationserfahrung aufwächst, die deutsche Staatsbürgerschaft hat (oder im Lauf der Zeit erhält). Menschen mit Migrationshintergrund der ,zweiten Generation', die wie ihre Eltern nicht die deutsche Staatsangehörigkeit besitzen, werden sich möglicherweise weniger stark der deutschen Gesellschaft verbunden

2 Bis 1993 als Aussiedlerinnen beziehungsweise Aussiedler bezeichnet, ab 1993 als Spätaussiedlerinnen beziehungsweise Spätaussiedler. fühlen und sich auch anteilig seltener freiwillig engagieren als jene Personen, die die deutsche Staatsbürgerschaft haben.

Schließlich ist zu fragen, welche Unterschiede sich zwischen der Vergleichsgruppe der Deutschen ohne Migrationshintergrund und den vier oben beschriebenen Gruppen hinsichtlich der Beteiligung am freiwilligen Engagement finden. Wir erwarten die geringsten Unterschiede zu der Vergleichsgruppe bei den Personen mit Migrationshintergrund, die der ,zweiten Generation' mit deutscher Staatsangehörigkeit angehören (also den Menschen mit Migrationshintergrund, die in Deutschland geboren sind und die deutsche Staatsangehörigkeit haben). Die größten Unterschiede zur Vergleichsgruppe erwarten wir bei Ausländerinnen und Ausländern mit eigener Zuwanderungserfahrung. Die Unterschiede zwischen Deutschen mit eigener Zuwanderungserfahrung und Deutschen ohne Migrationshintergrund sollten ebenfalls nicht sehr groß sein, weil in dieser Gruppe auch Personen sind, die sich einbürgern haben lassen, wofür unter anderem vorausgesetzt ist, seit mindestens acht Jahren in Deutschland zu leben. Es ist also sehr wahrscheinlich, dass diese Personengruppe mit der zivilgesellschaftlichen Landschaft in Deutschland vertraut ist. Für die gemeinschaftliche Aktivität, die das Mitmachen in gesellschaftlichen Bereichen bezeichnet, lassen sich dieselben ,hierarchisch ' abgestuften Erwartungen formulieren: Wer in Deutschland geboren ist und die deutsche Staatsangehörigkeit hat, wird ähnlich häufig außerhalb von Beruf und Familie irgendwo gemeinschaftlich und öffentlich mitmachen, ob mit Migrationshintergrund oder ohne. Wer aber im Ausland geboren ist und nicht die deutsche Staatsangehörigkeit hat, wird dies möglicherweise seltener tun. Die systematisch definierten Gruppen mit Migrationshintergrund spiegeln somit möglicherweise einen Gradienten beim Zugang zu Aktivität und Engagement wider.

Migration und sozioökonomischer Status: Die Differenzierung der Menschen mit Migrationshintergrund in vier Gruppen ist eine Vereinfachung, allerdings eine, die wir mit guten 
Gründen vorgenommen haben. Dennoch: Die vier Gruppen unterscheiden sich nicht allein mit Blick auf eigene Migrationserfahrung und Staatsbürgerschaft, sondern auch hinsichtlich weiterer Merkmale. Die in Deutschland geborenen Menschen mit Migrationshintergrund sind im Durchschnitt jünger als Menschen mit eigener Migrationserfahrung. Menschen mit eigener Migrationserfahrung, die keine deutsche Staatsangehörigkeit haben, verfügen anteilig häufiger über eine niedrigere Bildung als der Durchschnitt der Bevölkerung in Deutschland. Bei Deutschen mit eigener Migrationserfahrung ist dies anders: Sie verfügen anteilig häufiger über eine gute Schulbildung als der Durchschnitt der Bevölkerung in Deutschland. Unterschiede zwischen den vier Gruppen lassen sich also möglicherweise darauf zurückführen, dass bestimmte Merkmale in den unterschiedlichen Gruppen ungleich verteilt sind (Tucci 2013).

Es ist bekannt, dass freiwilliges Engagement durch sozioökonomische Ressourcen wie Bildung und Einkommen befördert wird (siehe Kapitel 16). Bei Bevölkerungsgruppen mit unterdurchschnittlicher Ressourcenausstattung ist also eine geringere zivilgesellschaftliche Beteiligung zu erwarten (Simonson, Hagen, Vogel \& Motel-Klingebiel 2013). Hierzu zählen im Durchschnitt Personen mit Migrationshintergrund, insbesondere jedoch Ausländerinnen und Ausländer sowie Deutsche mit eigener Zuwanderungserfahrung, die deutlich häufiger armutsgefährdet sind als Deutsche ohne Migrationshintergrund. Auch Qualifizierungslücken können eine Zugangsbarriere darstellen, etwa wenn Personen nie eine Schule besucht haben oder nicht lesen und schreiben können (Bundesamt für Migration und Flüchtlinge (BAMF) 2015) - dies betrifft besonders häufig Zuwanderinnen und Zuwanderer aus subsaharischen Staaten, die überwiegend eine eigene Migrationserfahrung, aber keine deutsche Staatsangehörigkeit haben (United Nations Educational, Scientific and Cultural Organization (UNESCO) 2015) oder keine Berufsausbildung abgeschlossen haben. Da zu erwarten ist, dass diese sozioökono- mischen Ressourcen innerhalb der vier Gruppen mit Migrationshintergrund und der Gruppe der Deutschen ohne Migrationshintergrund unterschiedlich verteilt sind, sollen die deskriptiven Gruppenvergleiche ergänzt werden durch Analysen, in denen für Geschlecht, Alter, Bildung, Einkommen und weitere Merkmale kontrolliert wird. Sollten die in den Gruppenvergleichen gefundenen Unterschiede in diesen multivariaten Analysen geringer ausfallen, so würde dies bedeuten, dass nicht allein der Migrationsstatus dafür verantwortlich ist, ob eine Person ein freiwilliges Engagement aufnimmt, sondern ihre mehr oder weniger begünstigte Lebenssituation. Sollten dagegen die Gruppenunterschiede auch in den multivariaten Analysen bestätigt werden, so würde dies die hohe Bedeutung der beiden Dimensionen hervorheben, die wir in diesem Kapitel zur Beschreibung der Vielfalt der Menschen mit Migrationshintergrund in Deutschland herangezogen haben: Eigene Migrationserfahrung und Staatsangehörigkeit.

Zielsetzung: In diesem Kapitel werden neben dem freiwilligen Engagement, der Engagementbereitschaft und den gemeinschaftlichen Aktivitäten auch die konkrete Ausgestaltung des Engagements in den Blick genommen, um Besonderheiten im freiwilligen Engagement von Personen mit Migrationshintergrund jeweils vergleichend $\mathrm{zu}$ den Deutschen ohne Migrationshintergrund herauszuarbeiten. Neben den erwarteten Unterschieden zwischen Menschen mit und ohne Migrationshintergrund sind auch zahlreiche Gemeinsamkeiten möglich, etwa bezüglich der gesellschaftlichen Engagementbereiche wie Sport und Bewegung, in denen ein hoher Anteil freiwillig Engagierter tätig ist oder bezüglich des Zeitaufwandes, der in das Engagement investiert wird. Zudem betrachten wir in diesem Kapitel die informelle Unterstützung im außerfamilialen sozialen Nahraum, die von Menschen mit Migrationshintergrund geleistet wird. Die Gegenüberstellung des öffentlichen Engagements und der privaten Unterstützung soll dazu genutzt werden, die gängige These zu überprüfen, ob sich Menschen mit Migrationshinter- 
grund lediglich seltener öffentlich engagieren, jedoch privaten Verpflichtungen in ihren sozialen Netzwerken anteilig häufiger nachkommen (siehe Kapitel 10). Begründet wird die Erwartung einer höheren privaten Einbindung etwa mit der Annahme von traditionelleren Einstellungen, die bestimmte Personengruppen mit Zuwanderungserfahrungen teilen, und mit der Annahme von größeren familialen und außerfamilialen sozialen Netzwerken dieser Personengruppen (Vogel 2012; Baykara-Krumme 2012). Um das freiwillige Engagement der in Deutschland lebenden Menschen mit Migrationshintergrund $\mathrm{zu}$ beschreiben, möchten wir in fünf Schritten vorgehen.

- Deskription der Personen mit Migrationshintergrund, die am Freiwilligensurvey 2014 teilgenommen haben: Im ersten Abschnitt soll die Gruppe der im Freiwilligensurvey vertretenen Personen mit Migrationshintergrund nach Art ihres Migrationshintergrundes dargestellt werden, um zu beschreiben, welches die häufigsten Geburtsländer beziehungsweise -regionen sind und in welcher Sprache die Befragten mit Migrationshintergrund jeweils am Interview teilgenommen haben.

- Freiwilliges Engagement, gemeinschaftliche Aktivität sowie Engagementbereitschaft: Im zweiten Abschnitt werden das freiwillige Engagement und die gemeinschaftliche Aktivität sowie die Bereitschaft untersucht, sich künftig $\mathrm{zu}$ engagieren, $\mathrm{um} \mathrm{zu}$ beantworten, ob sich Menschen mit Migrationshintergrund zu gleichen Anteilen zivilgesellschaftlich einbringen wie Menschen ohne Migrationshintergrund und welche Unterschiede sich nach Art des Migrationshintergrundes zwischen den Bevölkerungsgruppen zeigen.

- Ausgestaltung des freiwilligen Engagements: Im dritten Abschnitt steht die Ausgestaltung des freiwilligen Engagements bei Menschen mit Migrationshintergrund im Fokus - das heißt, in welchen Bereichen engagieren sich Menschen mit verschiedenem Migrationshintergrund, und unterscheiden sich diese von den Engagementbereichen der Menschen ohne Migrationshintergrund? Haben Engagierte mit Migrationshintergrund seltener eine Leitungsfunktion als Engagierte ohne Migrationshintergrund und verwenden sie unterschiedlich viel Zeit für ihre freiwilligen Tätigkeiten?

- Informelle Unterstützung: Im vierten Abschnitt wird die Ausübung informeller Unterstützung bezüglich Kinderbetreuung, Pflege und instrumentelle Hilfe bei Menschen mit Migrationshintergrund dargestellt, um neben dem häufig formal organisierten Engagement auch informelle Hilfeleistungen zu berücksichtigen, denen für die Integration sowie den gesellschaftlichen Zusammenhalt eine ebenso wichtige Funktion zukommen kann wie dem freiwilligen Engagement. Leisten Menschen mit Migrationshintergrund $\mathrm{zu}$ gleichen Anteilen informelle Unterstützung wie Menschen ohne Migrationshintergrund und welche Unterschiede zeigen sich nach Art des Migrationshintergrundes?

- Multivariate Analyse: Im fünften Abschnitt steht die Frage im Fokus, ob sich die Unterschiede im Engagement zwischen den verschiedenen Gruppen von Menschen mit Migrationshintergrund und Menschen ohne Migrationshintergrund auch dann zeigen, wenn die Heterogenität innerhalb der Gruppen (zum Beispiel nach Geschlecht, Alter, Bildung und Einkommen sowie nach weiteren Aspekten der Lebenssituation) berücksichtigt wird. Aus diesem Grund werden die deskriptiven Darstellungen ergänzt durch multivariate Analysen, in denen für weitere Merkmale kontrolliert wird. 


\subsection{Datengrundlage}

Die in diesem Kapitel berichteten Befunde basieren auf gewichteten Analysen. Für die Gewichtung wurden in allen Erhebungsjahren dieselben Gewichtungsmerkmale berücksichtigt. Dabei handelt es sich um die Designgewichtung, die sich auf die Auswahlwahrscheinlichkeit einer Person bezieht (Haushaltsgröße, Zahl der Festnetznummern und, nur für das Jahr 2014, auch die Zahl der Mobilfunknummern) und Gewichtungsmerkmale, die Abweichungen der Stichprobenverteilung von der Grundgesamtheit in bestimmten Dimensionen ausgleichen (Bundesland, Gemeindegrößenklassen, Geschlecht, Altersgruppen). Von einer Hinzunahme weiterer Gewichtungsmerkmale wie etwa des Bildungsstandes haben wir abgesehen, um die Vergleichbarkeit mit den bisher veröffentlichten Berichten zu erhalten.

Im Freiwilligensurvey wird freiwilliges Engagement erfasst als Arbeiten und Aufgaben, die außerhalb von Beruf und Familie sowie unentgeltlich oder gegen geringe Aufwandsentschädigung ausgeübt werden. Die öffentliche Aktivität wird als Mitmachen in vierzehn gesellschaftlichen Bereichen erfasst, ohne jedoch freiwillige oder ehrenamtliche Aufgaben oder Arbeiten zu übernehmen (siehe Kapitel 2 und Kapitel 3). Für freiwillig Engagierte liegen im Freiwilligensurvey eine Reihe von zusätzlichen Angaben dazu vor, wie die freiwilligen Tätigkeiten im Einzelnen ausgestaltet sind, zum Beispiel wieviel Zeit darauf pro Woche verwendet wird und ob im Engagement auch eine Leitungs- oder Vorstandfunktion übernommen wird (siehe Kapitel 2, Kapitel 11 und Kapitel 12). Wenn Engagierte mehrere freiwillige Tätigkeiten ausüben, beziehen sich die Ergebnisse zur Ausgestaltung des Engagements jeweils auf ihre zeitintensivste Tätigkeit. Für nicht-engagierte Personen liegen Angaben vor, ob sie bereit sind, sich künftig (sicher oder vielleicht) zu engagieren (siehe Kapitel 4).

Die außerfamilialen informellen Unterstützungsleistungen im sozialen Nahraum werden im Freiwilligensurvey anhand von drei Aspekten erfasst: Instrumentelle Hilfen für Nachbarinnen und Nachbarn, Freundinnen und Freunde sowie Bekannte und Andere; Kinderbetreuung bei nicht-eigenen Kindern; Pflege- und Betreuungstätigkeiten für nicht-verwandte Personen, die außerhalb des eigenen Haushaltes leben (siehe Kapitel 9 und Kapitel 10).

Um Personen mit Migrationshintergrund besser in den Freiwilligensurvey einzubeziehen, wurden 2014 erstmals Interviews nicht nur in deutscher Sprache geführt, sondern auch in fünf Fremdsprachen (Türkisch, Russisch, Polnisch, Englisch und Arabisch; siehe Kapitel 2). Wir betrachten im Folgenden fünf Bevölkerungsgruppen nach Art ihres Migrationshintergrundes: (1) Personen ohne deutsche Staatsangehörigkeit mit eigener Zuwanderungserfahrung; (2) Deutsche Staatsangehörige mit eigener Zuwanderungserfahrung; (3) Personen ohne deutsche Staatsangehörigkeit und ohne eigene Zuwanderungserfahrung; (4) Deutsche Staatsangehörige mit Migrationshintergrund, aber ohne eigene Zuwanderungserfahrung; (5) Deutsche Staatsangehörige ohne Migrationshintergrund. Die $\mathrm{Zu}$ ordnung der Befragten zu diesen fünf Kategorien erfolgt nach den vom Statistischen Bundesamt (2015b) festgelegten Kriterien - Staatsangehörigkeit, eigene Zuwanderungserfahrung nach 1949 beziehungsweise Zuwanderungserfahrung der Eltern - und basiert auf dem unten dargestellten Erhebungskonzept. ${ }^{3}$

Migrationserfahrung: Zunächst wird im Freiwilligensurvey 2014 geklärt, wo die befragte Person geboren ist:

Wo wurden Sie geboren?

3 Alle Analysen werden mit Blick auf das Jahr 2014 vorgenommen. Vergleiche mit früheren Wellen des Freiwilligensurveys werden in diesem Kapitel nicht durchgeführt, weil die Informationen, um den Migrationshintergrund einer Person differenziert klassifizieren zu können, in den früheren Wellen des Freiwilligensurveys nicht oder nur zum Teil vorliegen. 
Je nach Geburtsjahrgang stehen unterschiedliche Antwortkategorien zur Verfügung, diese wurden über die unten stehenden Altersgruppen gefiltert (Tabelle 23-1). Wenn eine befragte Person zum Beispiel 14 Jahre alt war, so wurden ihr diese Antwortkategorien genannt: Wo wurden Sie geboren? In den neuen Bundesländern? In den alten Bundesländern? Oder in einem anderen Land? Wenn eine befragte Person zum Zeitpunkt der Befragung im Jahr 2014 zum Beispiel 80 Jah- re alt war, wurden ihr diese Antwortkategorien genannt: In Deutschland, inklusive ehemaliger Ostgebiete, die heute nicht mehr zu Deutschland gehören? Oder in einem anderen Land? Sehr wenige Befragte des Freiwilligensurveys wollten ihr Alter nicht exakt angeben, aber sie haben sich auf Nachfrage in eine Altersstufe (kategorisiert) einordnen lassen. Auch diese Altersangabe wurde zur Filterführung der Antwortkategorien verwendet.

Tabelle 23-1: Antwortkategorien: Wo wurden Sie geboren?

\begin{tabular}{lll}
\hline Antwortkategorie & $\begin{array}{l}\text { Altersangabe der Befragten } \\
\text { (offen) }\end{array}$ & $\begin{array}{l}\text { Altersangabe der Befragten } \\
\text { (kategorisiert) }\end{array}$ \\
\hline $\begin{array}{l}\text { In Deutschland, inklusive ehemaliger } \\
\text { Ostgebiete, die heute nicht mehr } \\
\text { zu Deutschland gehören }\end{array}$ & 69 Jahre und älter & 65 Jahre und älter \\
\hline $\begin{array}{l}\text { In der sowjetischen Besatzungszone } \\
\text { In der amerikanischen, britischen }\end{array}$ & 65 bis 69 Jahre & - \\
\hline $\begin{array}{l}\text { oder französischen Besatzungszone } \\
\text { In der DDR }\end{array}$ & 65 bis 69 Jahre & - \\
\hline $\begin{array}{l}\text { In der BRD } \\
\text { In den neuen Bundesländern }\end{array}$ & 24 bis 65 Jahre & zwischen 21 und 64 Jahren \\
\hline In den alten Bundesländern & 24 bis 65 Jahre & zwischen 21 und 64 Jahren \\
\hline \begin{tabular}{l} 
In einem anderen Land \\
\hline
\end{tabular} & 14 bis 23 Jahre & zwischen 14 und 20 Jahren \\
\hline
\end{tabular}

Quelle: FWS 2014, eigene Darstellung (DZA).

Lautet die Antwort, In einem anderen Land, werden zwei Nachfragen gestellt:

Bitte nennen Sie mir Ihr Geburtsland. In welchem Jahr sind Sie nach Deutschland gezogen?

Eine eigene Zuwanderungserfahrung liegt vor, wenn jemand in einem anderen Land geboren und ab 1950 zugezogen ist.

Ein Migrationshintergrund in der zweiten Generation liegt vor, wenn die befragte Person in Deutschland geboren ist, jedoch mindestens ein Elternteil im Ausland geboren ist. Um dies festzustellen, werden allen Personen, die in
Deutschland geboren sind, noch weitere Fragen zum Geburtsland der Eltern gestellt:

Sind Ihre beiden Elternteile in Deutschland geboren? Wir meinen damit auch die ehemaligen Ostgebiete, die heute nicht mehr zu Deutschland gehören.

Antwortkategorien: ja, beide; ja, aber nur Ihre Mutter; ja, aber nur Ihr Vater; nein, beide nicht

Gegebenenfalls folgen die Fragen:

In welchem Land wurde Ihre Mutter geboren? In welchem Land wurde Ihr Vater geboren? 
Staatsangehörigkeit: Allen Befragten wird zudem eine Frage zur Staatsangehörigkeit gestellt, denn wer nicht die deutsche Staatsangehörigkeit besitzt beziehungsweise diese nicht seit seiner Geburt besitzt, wird ebenfalls als Person mit Migrationshintergrund betrachtet:

\section{Haben Sie die deutsche Staatsangehörigkeit?}

Wenn ja, folgen diese Zusatzfragen:

Haben Sie die deutsche Staatsangehörigkeit seit Geburt?

Und gegebenenfalls:

Wie haben Sie die deutsche Staatsangehörigkeit erworben?

Antwortkategorien: durch Einbürgerung; durch Anerkennung als Aussiedlerin/als Aussiedler; sonstiges

Zudem werden allen Befragten Fragen zur Staatsangehörigkeit der Eltern gestellt, da auch Personen, deren Eltern nicht die deutsche Staatsangehörigkeit haben (beziehungsweise hatten, falls sie bereits verstorben sind), als Personen mit Migrationshintergrund betrachtet werden:
Hat Ihre Mutter die deutsche Staatsangehörigkeit? Hat Ihr Vater die deutsche Staatsangehörigkeit?

Mit diesen Angaben kann die heterogene Gruppe der Menschen mit Migrationshintergrund differenziert beschrieben werden. Innerhalb der Gruppe der Personen mit Migrationshintergrund können im Freiwilligensurvey 2014 zudem weitere Gruppen zum Beispiel hinsichtlich ihres Geburtslandes beziehungsweise des Geburtslandes der Eltern unterschieden werden. Aufgrund der Vielzahl der verschiedenen Einwanderungshintergründe und der Vielzahl verschiedener Geburtsländer werden wir die Ergebnisse allein für die oben vorgestellten Gruppen mit Migrationshintergrund nach dem Konzept des Statistischen Bundesamtes darstellen und nicht nach den größten einzelnen Herkunfts- oder Sprachgruppen. Diese würden jeweils nur eine Minderheit der Personen mit Migrationshintergrund charakterisieren. Das Ziel des Freiwilligensurveys liegt jedoch in der Beschreibung der gesamten Wohnbevölkerung Deutschlands, die sich gerade durch eine Vielzahl von Personengruppen aus einer Vielzahl verschiedener Geburtsländer auszeichnet.

\subsection{Beschreibung der Personen mit Migrationshintergrund}

An der Erhebung des Deutschen Freiwilligensurveys 2014 haben 4.984 Personen mit Migrationshintergrund teilgenommen, das entspricht einem ungewichteten Anteil von 17,4 Prozent (gewichtet: 20,5 Prozent). Aus dem Mikrozensus ist bekannt, dass jeder beziehungsweise jede Fünfte in Deutschland einen Migrationshintergrund hat (Statistisches Bundesamt 2015). Aus dem Vergleich lässt sich schließen, dass Personen mit Migrationshintergrund im Freiwilligensurvey relativ gut abgebildet werden, obwohl sie zu etwas geringeren Anteilen am Interview teilge- nommen haben als Deutsche ohne Migrationshintergrund. Es sind insbesondere die Personen ohne deutsche Staatsangehörigkeit, die durch die Telefoninterviews weniger gut erreicht werden konnten, was häufig auf mangelnde deutsche Sprachkenntnisse zurückzuführen sein dürfte. Auch decken die fünf Fremdsprachen (Türkisch, Russisch, Polnisch, Englisch und Arabisch), in denen die Interviews 2014 erstmalig geführt wurden, lediglich einen Teil der Sprachen ab, die Migrantinnen und Migranten ohne deutsche Sprachkenntnisse sprechen (ohne diese fremd- 
sprachigen Interviews wäre die Untererfassung der Personen mit Migrationshintergrund in unserer Studie gravierender ausgefallen, siehe auch Abbildung 23-2). Es haben 1.485 Personen ohne deutsche Staatsangehörigkeit teilgenommen, das entspricht einem ungewichteten Anteil von 5,2 Prozent (gewichtet: 6,6 Prozent). In der Grundgesamtheit der Wohnbevölkerung Deutschlands beträgt der Anteil der Ausländerinnen und Ausländer 8,9 Prozent (siehe Kapitel 2).

Die Bevölkerung mit Migrationshintergrund ist heterogen zusammengesetzt und im Freiwilligensurvey wird dies auch abgebildet. Von den Befragten stellen deutsche Staatsangehörige mit Migrationshintergrund, aber ohne eigene Zuwanderungserfahrung mit 36,0 Prozent die größte Gruppe innerhalb aller Personen mit Migrationshintergrund. Diese Gruppe lässt sich kennzeichnen durch einen Migrationshintergrund der zweiten Generation. Die Personen, die dieser Gruppe angehören, sind meist nicht nur in Deutschland geboren, sondern haben hier in der Regel auch die Schule besucht und verfügen über entsprechende Deutschkenntnisse. Die deutsche Staatsangehörigkeit haben sie teils qua Geburt, teils durch Einbürgerung im späteren Lebensverlauf erhalten. In dieser Gruppe befinden sich viele jüngere Menschen (39,3 Prozent sind zwischen 14 und 29 Jahre alt) und vergleichsweise häufig handelt es sich bei ihnen um Personen, die noch zur Schule gehen (14,0 Prozent; Tabelle 23-2). Im
Vergleich zur Grundgesamtheit der in Deutschland lebenden Personen mit Migrationshintergrund ist diese Gruppe im Freiwilligensurvey überrepräsentiert (in der Grundgesamtheit stellt diese Gruppe 25,4 Prozent aller Menschen mit Migrationshintergrund, Statistisches Bundesamt 2015).

Die zweitgrößte Gruppe ist im Freiwilligensurvey mit 31,6 Prozent die Gruppe der deutschen Staatsangehörigen mit eigener $\mathrm{Zu}$ wanderungserfahrung. Diese Gruppe ist gekennzeichnet durch einen Migrationshintergrund der ersten Generation. Je nachdem, welches Lebensalter diese Personen zum Zeitpunkt der Zuwanderung erreicht hatten, haben sie die Schule im Herkunftsland oder aber in Deutschland besucht. Viele von ihnen haben als, ethnisch Deutsche in ihren Herkunftsländern bereits Deutsch gelernt, bevor sie als (Spät-)Aussiedlerinnen und Aussiedler nach Deutschland gekommen sind. Personen im mittleren Erwachsenenalter mit Migrationshintergrund sind in dieser Gruppe überproportional häufig vertreten (45,8 Prozent dieser Gruppe sind zwischen 30 und 49 Jahre alt, 24,5 Prozent zwischen 50 und 64 Jahre) (Tabelle 23-2). Auch diese Gruppe ist im Freiwilligensurvey überrepräsentiert, allerdings nur leicht (in der Grundgesamtheit stellt diese Gruppe 30,6 Prozent aller Menschen mit Migrationshintergrund, Statistisches Bundesamt 2015). 
Tabelle 23-2: Beschreibung der Bevölkerungsgruppen nach Geschlecht, Alter und Bildung 2014

\begin{tabular}{lccccc}
\hline & $\begin{array}{c}\text { Ohne Migrations- } \\
\text { hintergrund }\end{array}$ & \multicolumn{2}{c}{$\begin{array}{c}\text { Ohne eigene } \\
\text { Zuwanderungserfahrung } \\
\text { mit Migrationshintergrund }\end{array}$} & \multicolumn{2}{c}{$\begin{array}{c}\text { Mit eigener } \\
\text { Zuwanderungserfahrung }\end{array}$} \\
\hline & $\begin{array}{c}\text { Deutsche } \\
\text { ohne Migrations- } \\
\text { hintergrund }\end{array}$ & $\begin{array}{c}\text { Personen } \\
\text { mit deutscher } \\
\text { Staatsan- } \\
\text { gehörigkeit }\end{array}$ & $\begin{array}{c}\text { Personen } \\
\text { ohne deutsche } \\
\text { Staatsan- } \\
\text { gehörigkeit }\end{array}$ & $\begin{array}{c}\text { Personen } \\
\text { mit deutscher } \\
\text { Staatsan- } \\
\text { gehörigkeit }\end{array}$ & $\begin{array}{c}\text { Personen } \\
\text { ohne deutsche } \\
\text { Staatsan- } \\
\text { gehörigkeit }\end{array}$ \\
\hline Frauen & 51,1 & 53,2 & 47,3 & 53,6 & 49,6 \\
\hline Männer & 48,9 & 46,9 & 52,7 & 46,4 & 50,4 \\
\hline 14 bis 29 Jahre & 18,0 & 39,3 & 47,6 & 19,5 & 23,5 \\
\hline 30 bis 49 Jahre & 29,0 & 27,5 & 41,4 & 45,8 & 52,6 \\
\hline 50 bis 64 Jahre & 26,3 & 19,8 & 4,8 & 24,5 & 15,8 \\
\hline 65 Jahre und älter & 26,8 & 13,4 & 6,2 & 10,2 & 8,2 \\
\hline Noch Schüler/in & 3,9 & 14,0 & 15,9 & 2,9 & 2,5 \\
\hline Niedrige Bildung & 23,5 & 19,7 & 21,3 & 14,3 & 14,4 \\
\hline Mittlere Bildung & 32,0 & 26,5 & 31,7 & 31,2 & 28,8 \\
\hline Hohe Bildung & 40,6 & 39,7 & 31,2 & 51,6 & 54,3 \\
\hline
\end{tabular}

Quelle: FWS 2014, gewichtet, eigene Berechnungen (DZA). Basis: Alle Befragten $(n=28.690)$.

Dargestellt sind Spaltenprozente (die Prozentzahlen innerhalb einer Spalte summieren sich pro Differenzierungskriterium (Geschlecht, Alter, Bildung) zu 100 Prozent, Abweichungen sind rundungsbedingt.

Die Gruppe der Personen mit eigener Zuwanderungserfahrung, die ausschließlich die Staatsangehörigkeit eines anderen Landes haben, stellt 26,5 Prozent aller Personen mit Migrationshintergrund im Freiwilligensurvey. Auch bei ihnen ist das Lebensalter bei Zuwanderung ausschlaggebend für die schulische und berufliche Integration in Deutschland. Über die Hälfte der Personen dieser Gruppe verfügt über ein hohes Bildungsniveau und mehr als jede zweite Person ist im Alter zwischen 30 und 49 Jahren. Diese Gruppe ist im Freiwilligensurvey unterrepräsentiert (in der Grundgesamtheit stellt diese Gruppe 35,8 Prozent aller Menschen mit Migrationshintergrund).

Die kleinste Gruppe im Freiwilligensurvey mit 6,0 Prozent ist die Gruppe der ausländischen Staatsangehörigen ohne eigene Zuwanderungserfahrung. Die Angehörigen dieser Gruppe haben in der Regel die Schule in Deutschland besucht oder besuchen sie noch - 15,9 Prozent dieser Gruppe sind Schülerinnen und Schüler. Die deutsche Staatsangehörigkeit zu erhalten wäre für sie möglich, wenn sie die Bedingungen zur Einbürgerung erfüllen und einen entsprechenden Antrag stellen. ${ }^{4}$ Diese Gruppe ist im Freiwilligensurvey ebenfalls leicht unterrepräsentiert (in der Grundgesamtheit der Wohnbevölkerung Deutschlands mit Migrationshintergrund stellt diese Gruppe 8,2 Prozent aller Menschen mit Migrationshintergrund).

4 Eingebürgert werden kann, wer in Deutschland lebt und seinen Unterhalt bestreiten kann. Nicht eingebürgert wird, wer über keine ausreichenden deutschen Sprachkenntnisse verfügt oder straffällig geworden ist. Bei Einbürgerung muss die alte Staatsangehörigkeit aufgegeben werden (Lämmermann 2013). 
Diese vier Gruppen von Personen mit Migrationshintergrund lassen sich auch danach charakterisieren, welches Geburtsland die Personen (bei eigener Zuwanderungserfahrung) beziehungsweise welches Geburtsland ihre Eltern haben. Die im Freiwilligensurvey 2014 Befragten, die nach Deutschland zugewandert sind, kommen aus über 120 verschiedenen Ländern. Wir betrachten hier drei Geburtsregionen im Detail, aus denen jeweils besonders viele Einwanderinnen und Einwanderer stammen: Die ehemalige Sowjetunion beziehungsweise deren Nachfolgestaaten, die sich in der Gemeinschaft Unabhängiger Staaten (GUS) zusammengeschlossen haben, sowie Polen und die Türkei. ${ }^{5}$

\section{Abbildung 23-1: Geburtsregionen nach Art des Migrationshintergrundes 2014}

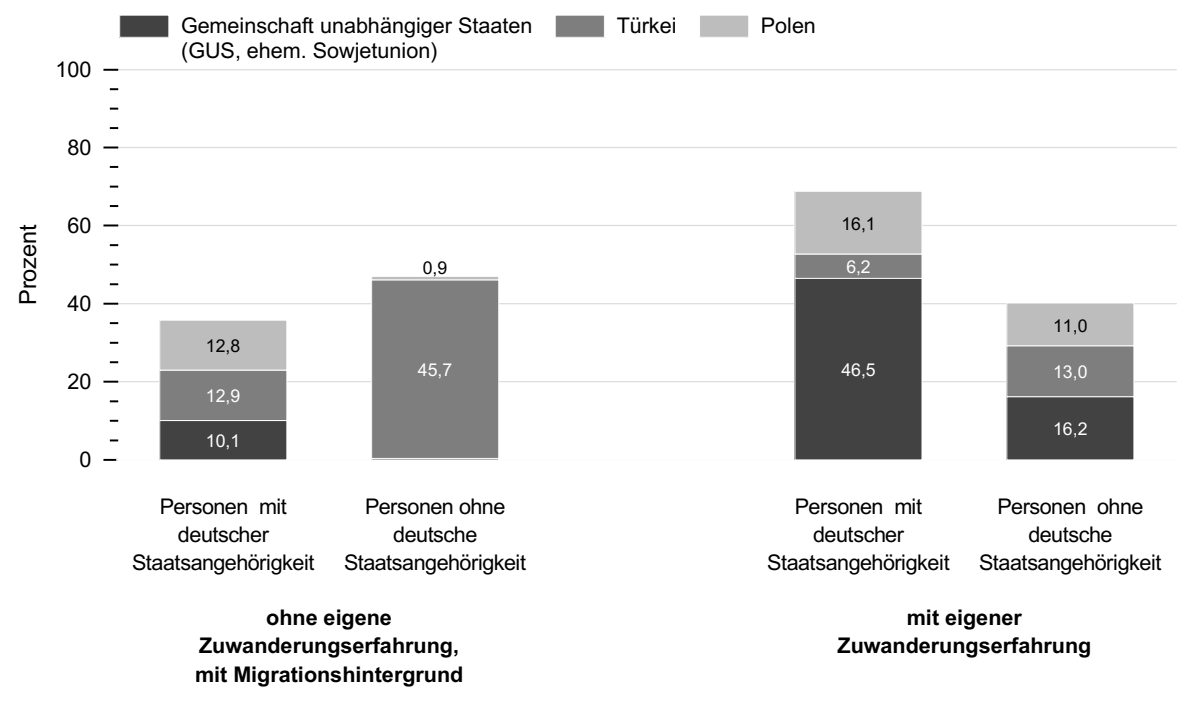

Quelle: FWS 2014, gewichtet, eigene Berechnungen (DZA). Basis: Alle Befragten mit Migrationshintergrund $(n=4.984)$. Zu 100 fehlende Prozente: Andere Geburtsregion.

Welche der vier Gruppen mit Migrationshintergrund ist nun geprägt durch welche Geburtsländer beziehungsweise -regionen? Die Gruppe der deutschen Staatsangehörigen mit eigener Zuwanderungserfahrung ist sehr deutlich geprägt durch Personen aus Geburtsländern, die heute zur Gemeinschaft Unabhängiger Staaten (GUS) gehören (46,5 Prozent aller deutschen Staatsangehörigen mit eigener Zuwanderungserfahrung wurden dort geboren) sowie durch das Geburtsland Polen (16,1 Prozent aller deutschen Staatsangehörigen mit eigener Zuwanderungserfahrung wurden in Polen geboren; Abbildung 23-1). Von den deutschen Staatsangehörigen mit eigener Zuwanderungserfahrung im Alter von
14 bis 29 Jahren stammen sogar 61,0 Prozent aus der GUS sowie weitere 10,3 Prozent aus Polen (Tabelle 23-1 im Online-Anhang). Diese Gruppe der deutschen Staatsangehörigen mit eigener Zuwanderungserfahrung ist somit geprägt durch (Spät-)Aussiedlerinnen und (Spät-)Aussiedler, die als Angehörige deutscher Minderheiten in

5 Die Gruppen von Personen mit jeweiligem Migrationshintergrund aus Polen oder der ehemaligen Sowjetunion sind in der Stichprobe sehr gut abgebildet, die türkeistämmige Gruppe weniger gut, trotz der Möglichkeit, die Befragung auf Türkisch durchzuführen (siehe Kapitel 2). Die Gründe dafür, warum türkeistämmige Personen seltener an Telefonbefragungen teilnehmen, sind vielfältig (Baykara-Krumme 2010). 
der ehemaligen Sowjetunion und in verschiedenen östlichen Staaten Mitteleuropas direkt bei Einreise nach Deutschland die deutsche Staatsangehörigkeit erhalten haben. Diese Gruppe der Deutschen mit eigener Zuwanderungserfahrung lässt sich von allen Gruppen mit Migrationshintergrund am besten durch die betrachteten Geburtsregionen charakterisieren, nur ein Drittel sind in einem anderen Land geboren und nicht auf dem Gebiet der heutigen GUS, Polens oder der Türkei. Zudem umfasst diese Gruppe Personen, die die deutsche Staatsangehörigkeit durch Einbürgerung erhalten haben.

Die Gruppe der ausländischen Staatsangehörigen mit eigener Zuwanderungserfahrung ist bezüglich der jeweiligen Geburtsländer deutlich heterogener: 16,2 Prozent dieser Gruppe sind in der ehemaligen Sowjetunion beziehungsweise in einem der Staaten geboren, die sich zur GUS zusammengeschlossen haben, 11,0 Prozent in Polen und weitere 13,0 Prozent in der Türkei (Abbildung 23-1).

Die Gruppe der ausländischen Staatsangehörigen ohne eigene Zuwanderungserfahrung ist hingegen sehr deutlich geprägt durch das Geburtsland Türkei: 45,7 Prozent der ausländischen Staatsangehörigen ohne eigene Zuwanderungserfahrung haben in der Türkei geborene Eltern und somit sehr wahrscheinlich Eltern, die die türkische Staatsangehörigkeit haben. Obwohl die Befragten selbst in Deutschland geboren wurden, haben sie nicht die deutsche Staatsangehörigkeit, weil diese nach dem Abstimmungsprinzip vergeben wird: Ein Kind erhält die deutsche Staatsangehörigkeit bei Geburt nur dann, wenn die Mutter oder der Vater oder beide deutsche Staatsangehörige sind. In der jüngsten Altersgruppe der 14- bis 29-Jährigen hat sogar mehr als die Hälfte der ausländischen Staatsangehörigen ohne eigene Zuwanderungserfahrung mindestens ein türkisches Elternteil (Tabelle 23-1 im Online-Anhang). Für einen Großteil dieser Personengruppe besteht im Übrigen die Möglichkeit, die deutsche Staatsangehörigkeit zu beantragen, allerdings haben sich einige dagegen entschieden, gegebenenfalls auch um nicht ihre alte Staatsangehörigkeit aufgeben zu müssen. ${ }^{6}$

In der Gruppe der deutschen Staatsangehörigen ohne eigene Zuwanderungserfahrung haben lediglich 12,9 Prozent mindestens ein türkisches Elternteil. Wiederum ist dieser Anteil in der Altersgruppe der 14- bis 29-Jährigen mit 21,8 Prozent am höchsten (Tabelle 23-1 im Online-Anhang). Die Gruppe der deutschen Staatsangehörigen ohne eigene Zuwanderungserfahrung lässt sich am wenigsten gut durch die drei Geburtsregionen GUS, Polen und Türkei charakterisieren: Insgesamt haben knapp zwei Drittel dieser Gruppe Eltern mit einem anderen Geburtsland (Abbildung 23-1).

Zwei vergleichsweise große Gruppen sind somit russischsprachige Personen - von den deutschen Staatsangehörigen mit eigener $\mathrm{Zu}$ wanderungserfahrung stammt fast jeder Zweite aus der ehemaligen Sowjetunion beziehungsweise ihren Nachfolgestaaten -, und türkeistämmige Personen - von den ausländischen Staatsangehörigen ohne eigene Zuwanderungserfahrung sind knapp die Hälfte türkeistämmig. Aufgrund von Anwerbeabkommen sind nicht nur aus der Türkei (seit 1961) sogenannte,Gastarbeiter' nach Deutschland eingewandert, sondern auch aus Italien (seit 1955), Spanien und Griechenland (seit 1960) sowie weiteren Ländern wie Portugal und Jugoslawien, deren Familien häufig noch heute in Deutschland leben. Viele Arbeitsmigrantinnen und Arbeitsmigranten stammen zum Beispiel aus Polen und weiteren osteuropäischen EU-Staaten. Nach türkischen und italienischen Personen sind die in Deutschland lebenden polnischen Staatsangehörigen die drittgrößte Nationalitätengruppe (Haug 2013). Die Mehrheit der (Spät-)Aussiedlerinnen und (Spät-)Aussiedler ist neben deutsch- insbesondere russischsprachig und stammt aus der ehemaligen Sowjetunion, aber auch aus osteuropäischen Staaten wie Polen und der Slowakei.

$6 \mathrm{Zu}$ den verschiedenen Aspekten der Mehrstaatigkeit siehe Bundesamt für Migration und Flüchtlinge (BAMF) 2016. 
Abbildung 23-2: Interviewsprache nach Art des Migrationshintergrundes 2014

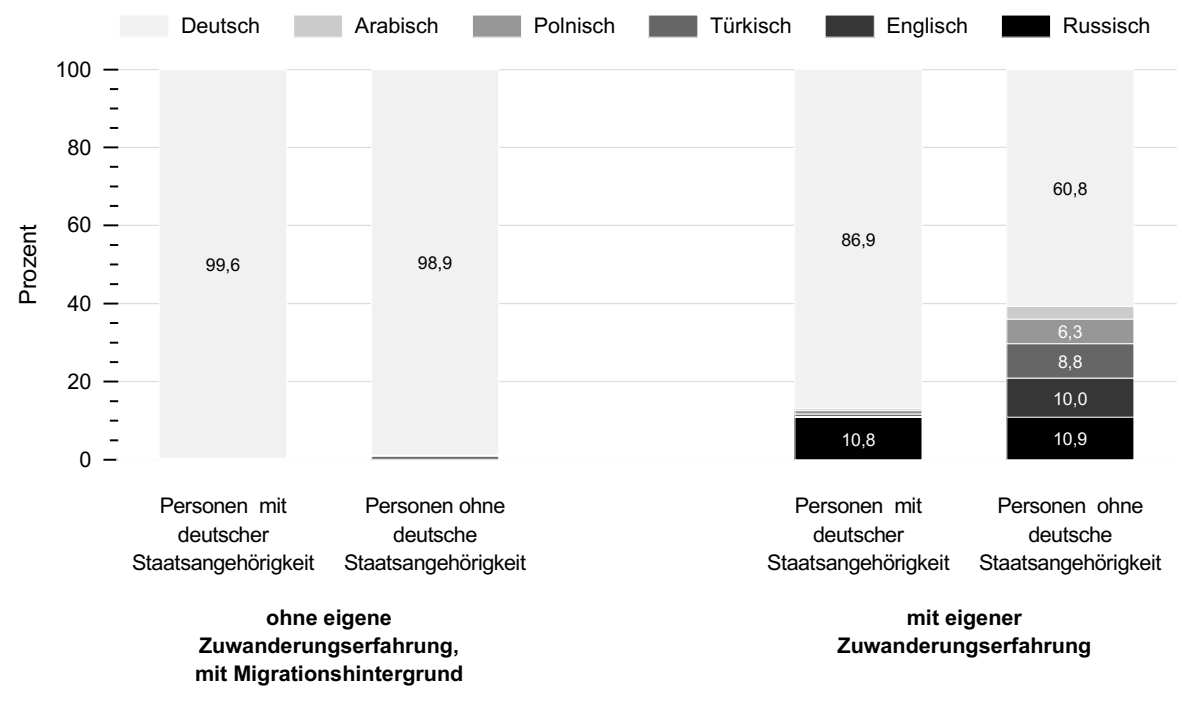

Quelle: FWS 2014, gewichtet, eigene Berechnungen (DZA). Basis: Alle Befragten mit Migrationshintergrund $(n=4.984)$.

Alle Personen ohne Migrationshintergrund und mehr als jede vierte von fünf Personen mit Migrationshintergrund haben das Freiwilligensurvey-Telefoninterview auf Deutsch mitgemacht. In den vier Gruppen mit Migrationshintergrund haben die Personen zudem unterschiedlich häufig in einer Fremdsprache am Telefoninterview teilgenommen. ${ }^{7}$ Insbesondere von Personen mit eigener Zuwanderungserfahrung wurde dieses Angebot angenommen, während Deutsche und Personen ohne deutsche Staatsangehörigkeit, aber ohne eigene Zuwanderungserfahrung in der Regel über ausreichende
Deutschkenntnisse verfügen. Personen ohne deutsche Staatsangehörigkeit, die nach Deutschland eingewandert sind, konnten zu etwa gleichen Teilen durch die Fremdsprachen Russisch, Türkisch und Englisch erreicht werden, zudem fanden Interviews auf Polnisch und Arabisch statt (Abbildung 23-2). Deutsche mit eigener $\mathrm{Zu}-$ wanderungserfahrung wurden neben Deutsch hauptsächlich durch Russisch erreicht, was plausibel ist, da es sich überwiegend um (Spät-)Aussiedlerinnen und (Spät-)Aussiedler handelt, während die anderen Fremdsprachen für diese Gruppe kaum eine Rolle spielten.

7 Es wurden 705 fremdsprachige Interviews geführt, das entspricht 2,5 Prozent aller geführten Interviews, davon 326 auf Russisch, 115 auf Türkisch, 88 auf Polnisch, 47 auf Arabisch und 129 auf Englisch, zudem wurden 27.985 deutschsprachige Interviews geführt, dies entspricht 97,5 Prozent aller Interviews (siehe Kapitel 2). 


\subsection{Engagement, Aktivität und Engagementbereitschaft nach Migrationshintergrund}

\subsubsection{Freiwilliges Engagement und gemeinschaftliche öffentliche Aktivität}

Von allen Personen mit Migrationshintergrund engagieren sich 31,5 Prozent (Abbildung 23-3). Sie engagieren sich somit anteilig deutlich seltener freiwillig als Personen ohne Migrationshintergrund (46,8 Prozent). Der Anteil Engagierter unterscheidet sich aber stark nach der Art des Migrationshintergrundes (Abbildung 23-3). In der Gruppe der Personen mit Migrationshintergrund der zweiten Generation, die die deutsche Staatsangehörigkeit besitzen, ist der Anteil En- gagierter mit 43,2 Prozent ähnlich hoch wie bei Personen ohne Migrationshintergrund. Deutlich geringer ist der Anteil engagierter Personen in der Gruppe der Personen mit Migrationshintergrund der zweiten Generation ohne deutsche Staatsangehörigkeit: Hier beträgt die Engagementquote 31,1 Prozent. Am geringsten ist der Anteil bei ausländischen Staatsangehörigen mit eigener Zuwanderungserfahrung: Lediglich 21,7 Prozent von ihnen sind freiwillig engagiert; Auch bei deutschen Staatsangehörigen mit eigener Zuwanderungserfahrung ist der Anteil der Engagierten mit einem guten Viertel unterdurchschnittlich.

\section{Abbildung 23-3: Anteile Engagierter und Anteile Aktiver nach Art des Migrationshintergrundes 2014}

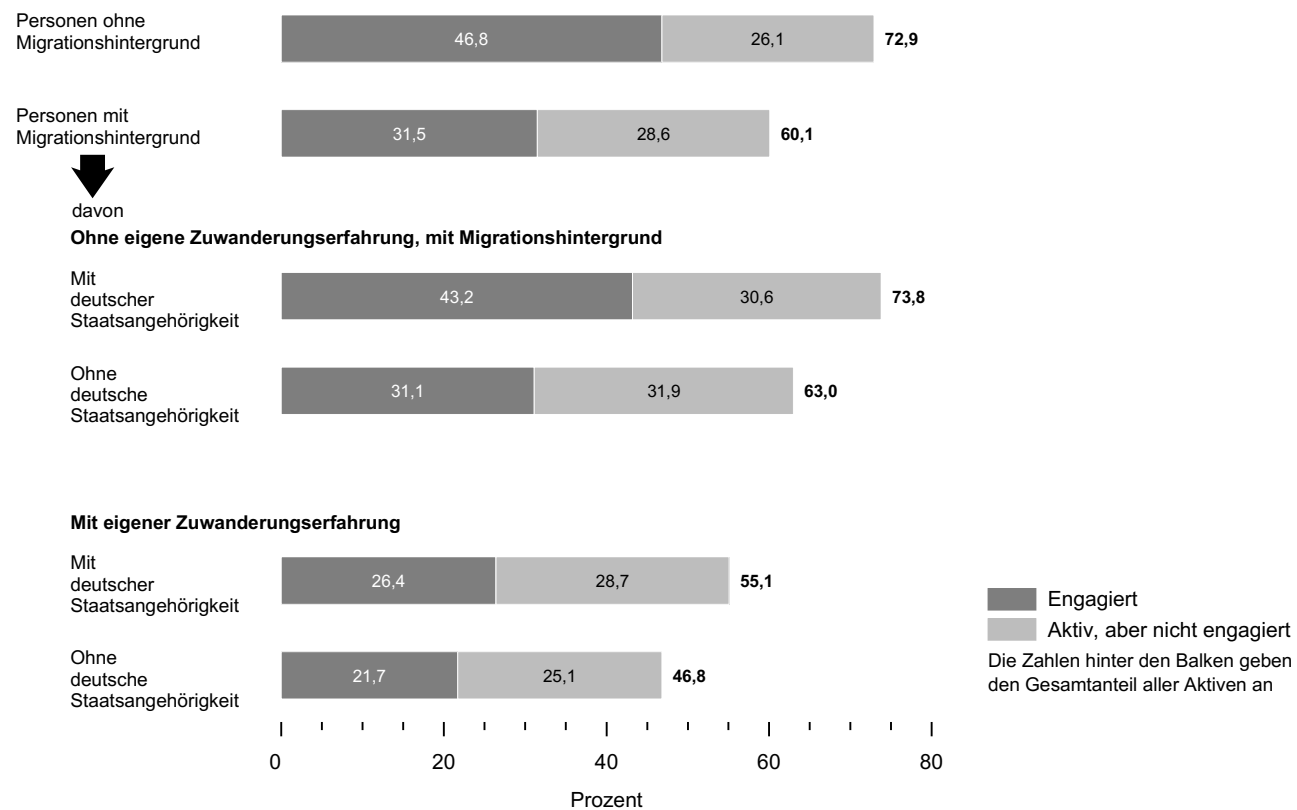

Quelle: FWS 2014, gewichtet, eigene Berechnungen (DZA). Basis: Alle Befragten ( $n=28.689$ ).

Festzuhalten ist, dass insbesondere Personen mit eigener Zuwanderungserfahrung geringere
Engagementquoten aufweisen, sowohl mit deutscher, aber insbesondere auch ohne deutsche 
Staatsangehörigkeit. Die eigene Zuwanderungserfahrung, die im Großteil der Fälle mit dem zumindest zeitweiligen Schulbesuch im Ausland und einer entsprechenden Sozialisationserfahrung einhergeht, scheint hierfür ebenso bedeutsam wie die Migrationserfahrung in Deutschland, die teils mit Integrationsschwierigkeiten wie Arbeitslosigkeit und Armutserfahrungen einhergeht, teils auch einfach mit Unkenntnis der zivilgesellschaftlichen Akteure und der Gelegenheiten für freiwilliges Engagement im Aufnahmeland Deutschland.

Gemeinschaftlich aktiv ist ein weiteres Viertel aller Personen ohne Migrationshintergrund (Abbildung 23-3). Dieser Anteil der aktiven, aber nicht-engagierten ist bei Personen mit Migrationshintergrund mit 28,6 Prozent sogar etwas höher als bei Personen ohne einen Migrationshintergrund. Da Aktivität durchaus als Vorstufe von Engagement betrachtet werden kann, gibt dieses Ergebnis einen Hinweis darauf, dass Menschen mit Migrationshintergrund durchaus zivilgesellschaftlich aktiv sind und von Akteuren in den Organisationen und Einrichtungen auch für die Übernahme von Aufgaben oder Arbeiten gewonnen werden könnten.

Insgesamt ist der Anteil von Menschen mit Migrationshintergrund, die öffentlich aktiv (einschließlich freiwillig engagiert) sind, mit unter zwei Dritteln allerdings geringer als bei Menschen ohne Migrationshintergrund, von denen sind fast drei Viertel aktiv. Dabei kann öffentlich aktiv zu sein als eine wichtige Bedingung dafür angesehen werden, Zugang zu einer freiwilligen Tätigkeit zu finden (siehe Kapitel 3). Von allen Gruppen mit Migrationshintergrund sind die Ausländerinnen und Ausländer mit eigener Zuwanderungserfahrung zu den geringsten Anteilen aktiv beteiligt, weniger als die Hälfte von ihnen sind öffentlich gemeinschaftlich aktiv (Abbildung 23-3). Da es sich hierbei um die erste Generation von Zuwanderinnen und Zuwanderer in Deutschland handelt, die zum Teil schon über 40 Jahre in Deutschland leben, deutet dies darauf hin, dass die zivilgesellschaftliche Integration für diese Generation bislang nicht uneingeschränkt gelungen ist und die Teilhabefunktion des freiwilligen Engagements für diese Gruppe nur eingeschränkt zur Verfügung steht. Möglicherweise liegt das mit daran, dass bei den Arbeitsmigrantinnen und Arbeitsmigranten eine Rückkehrneigung in ihr Herkunftsland unterstellt wurde, die sich allerdings nicht manifestierte, auch nicht im Ruhestandsalter, das viele Arbeitsmigrantinnen und Arbeitsmigranten heute bereits erreicht haben. Auch verfügen manche Personen aus dieser ersten Generation nach wie vor nur über geringe Deutschkenntnisse. Dieses Ergebnis sagt jedoch nichts darüber aus, wie gelungen die Integration in anderen Lebens- und Gesellschaftsbereichen ist, und schließlich ist auch die Mehrheit der Deutschen ohne Migrationshintergrund nicht freiwillig engagiert.

Hervorzuheben ist in der gemeinsamen Betrachtung von Engagement und Aktivität zudem, dass wir im direkten Vergleich der beiden Gruppen der Deutschen ohne Migrationshintergrund und der Deutschen mit Migrationshintergrund, aber ohne eigene Zuwanderungserfahrung kaum einen Unterschied im Anteil Aktiver sehen, aber Letztere (Deutsche mit Migrationshintergrund) einen etwas geringeren Anteil Engagierter aufweisen (43,2 Prozent). Das bedeutet, in der zweiten Generation, die in Deutschland geboren und hier zur Schule gegangen ist, und die die deutsche Staatsangehörigkeit hat, sehen wir keinen Unterschied in der Aktivität, aber einen geringfügigen Unterschied im Engagement. Zudem ist der Anteil freiwillig Engagierter nochmals geringer bei Personen ohne deutsche Staatsangehörigkeit und ohne eigene Zuwanderungserfahrung, obwohl sie ganz überwiegend in Deutschland zur Schule gegangen sein dürften. Dass die deutschen Staatsangehörigen mit Migrationshintergrund der zweiten Generation in ihrem Verhaltensmuster der Gruppe der Deutschen ohne Migrationshintergrund am ähnlichsten sind, gilt insbesondere auch dann, wenn wir die Anteile differenziert nach Geschlecht und nach Altersgruppen betrachten.

Es zeigen sich folgende Geschlechterunterschiede bezüglich des freiwilligen Engagements 
von Menschen mit Migrationshintergrund. Sowohl bei Personen mit Migrationshintergrund als auch ohne Migrationshintergrund weisen Frauen einen geringeren Anteil an Engagierten auf. Bei Deutschen ohne Migrationshintergrund sind 44,3 Prozent der Frauen und 49,4 Prozent der Männer freiwillig engagiert (Tabelle 23-3a im Online-Anhang). Bei Personen mit Migrationshintergrund ist die Geschlechterdifferenz im Engagement weniger ausgeprägt: 31,2 Prozent der Frauen und 31,8 Prozent der Männer mit Migrationshintergrund engagieren sich freiwillig. Nach Art des Migrationshintergrundes zeigen sich weitere Geschlechterunterschiede: In der Gruppe der Personen ohne deutsche Staatsangehörigkeit mit eigener Zuwanderungserfahrung ist der Anteil Engagierter bei Frauen ebenfalls etwas niedriger als bei Männern (21,2 Prozent zu 22,2 Prozent; Tabelle 23-3b im Online-Anhang). Ebenso zeigt sich in der Gruppe der Deutschen ohne eigene Zuwanderungserfahrung eine niedrigere Engagementquote bei den Frauen (41,0 Prozent) als bei den Männern (45,7 Prozent). Sowohl bei den Deutschen mit eigener Zuwanderungserfahrung als auch bei den ausländischen Staatsangehörigen ohne eigene Zuwanderungserfahrung liegt der Anteil der Engagierten bei Frauen jedoch über dem der Männer. 27,5 Prozent der Frauen und 25,1 Prozent der Männer mit deutscher Staatsangehörigkeit und eigener Zuwanderungserfahrung sind engagiert; 33,4 Prozent der Frauen und 29,0 Prozent der Männer mit ausländischer Staatsangehörigkeit und ohne eigene Zuwanderungserfahrung sind engagiert.

Darüber hinaus zeigt sich nach Altersgruppen, dass der Anteil Engagierter bei den Personen im Alter von 65 und mehr Jahren jeweils am geringsten ist. Lediglich 34,9 Prozent der Deutschen ohne Migrationshintergrund dieser Altersgruppe engagieren sich (gesamt 46,8 Prozent) sowie 26,2 Prozent der Personen mit Migrationshintergrund (gesamt 31,5 Prozent; Tabelle 23-3b im Online-Anhang). Für die drei Arten des Migrationshintergrundes, für die wir die Altersdifferenzierung betrachten (bei der Gruppe der Personen ohne deutsche Staatsangehörigkeit und ohne eigene Zuwanderungserfahrung ist die Fallzahl für eine Altersdifferenzierung in den höheren beiden Altersgruppen zu gering) zeigt sich dasselbe Muster (Tabelle 23-3a im Online-Anhang). Am häufigsten engagieren sich Personen im Alter von 14 bis 29 Jahren beziehungsweise im Alter von 30 bis 49 Jahren, je nach Gruppe, die betrachtet wird, in unterschiedlicher Reihenfolge.

In allen Bevölkerungsgruppen zeigt sich darüber hinaus ein starker Bildungsgradient im Anteil freiwillig Engagierter, allerdings auf sehr unterschiedlichem Niveau. Von allen Personen mit Migrationshintergrund engagieren sich nur 17,7 Prozent derjenigen mit niedriger Bildung freiwillig, aber 35,7 Prozent derjenigen mit hoher Bildung (Tabelle 23-3c im Online-Anhang). Bei den Deutschen ohne Migrationshintergrund betragen die entsprechenden Anteile 30,2 Prozent bei denjenigen mit niedriger Bildung und 57,3 Prozent bei denjenigen mit hoher Bildung. Am geringsten sind die Anteile freiwillig Engagierter bei den niedriggebildeten Personen ohne deutsche Staatsangehörigkeit mit eigener Zuwanderungserfahrung (Tabelle 23-3d im Online-Anhang): Lediglich 11,9 Prozent von ihnen sind freiwillig engagiert. Auch bei den Deutschen mit eigener Zuwanderungserfahrung und geringer Bildung ist die Engagementquote mit 14,3 Prozent sehr niedrig. Engagementförderung scheint hier erstrebenswert, insbesondere unter dem Gesichtspunkt, dass Engagement häufig Lernerfahrungen ermöglicht, die auch für andere Lebensbereiche von Nutzen sein können (siehe Kapitel 13).

\subsubsection{Engagementbereitschaft}

Hinsichtlich der Engagementbereitschaft übertreffen nicht-engagierte Personen mit Migrationshintergrund jene ohne Migrationshintergrund: 13,6 Prozent der Personen mit Migrationshintergrund antworten, sie sind ,sicher' bereit, sich künftig engagieren zu wollen, im Vergleich zu 10,8 Prozent bei Personen ohne Migrationshintergrund (Abbildung 23-4). Men- 
schen mit Migrationshintergrund sind zudem anteilig wesentlich häufiger ,vielleicht ' bereit, sich zu engagieren (54,5 Prozent im Vergleich zu 45,0 Prozent bei Personen ohne Migrationshintergrund). Hier liegt ein Potenzial für zivilgesellschaftliche Organisationen, neue Engagierte zu gewinnen (siehe Kapitel 4). Die Differenzen in der Engagementbereitschaft zwischen Menschen mit beziehungsweise ohne Migrationshintergrund sind dabei besonders ausgeprägt bei
Personen mit niedriger Bildung: 45,8 Prozent der niedriggebildeten Personen mit Migrationshintergrund geben an, dass sie vielleicht bereit sind, sich zukünftig zu engagieren (10,7 Prozent sind sicher bereit sich zukünftig zu engagieren). Bei den Niedriggebildeten ohne Migrationshintergrund sind dagegen nur 29,2 Prozent vielleicht und 6,0 Prozent sicher bereit (Tabelle $23-4 \mathrm{c}$ im Online-Anhang).

\section{Abbildung 23-4: Engagementbereitschaft nach Art des Migrationshintergrundes 2014}

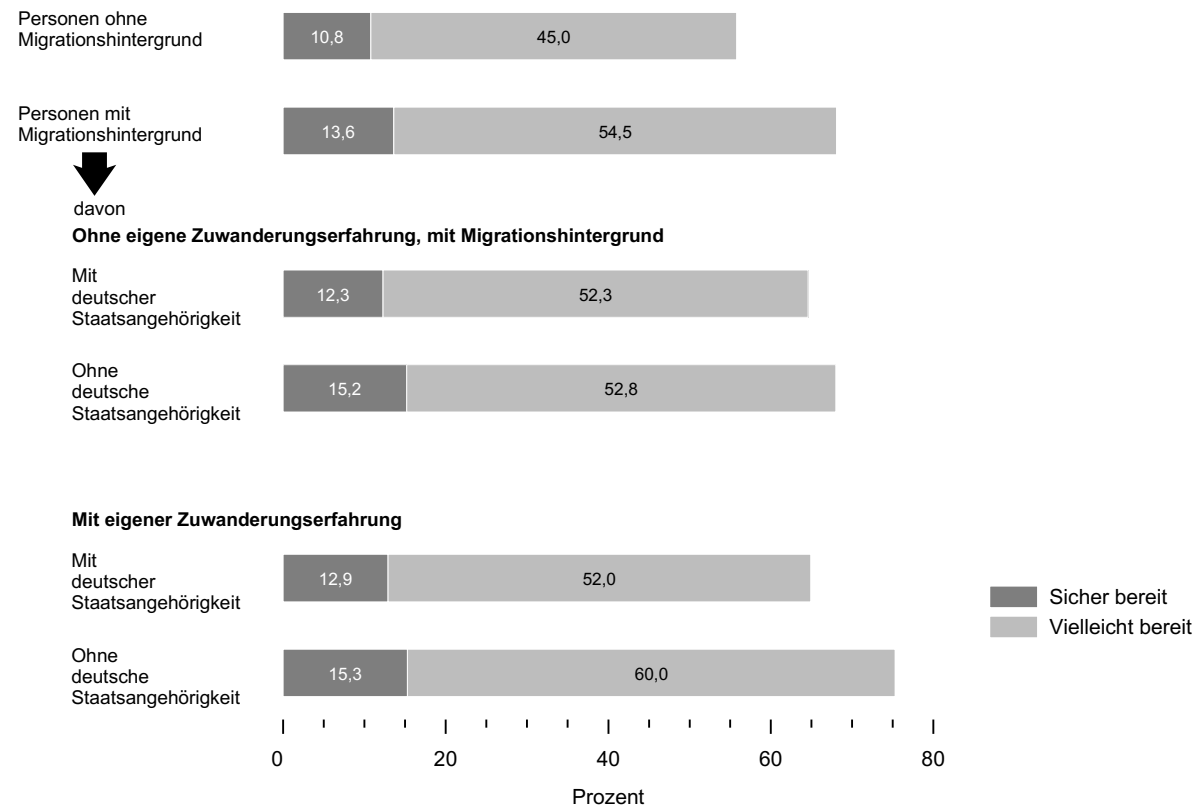

Quelle: FWS 2014, gewichtet, eigene Berechnungen (DZA). Basis: Alle Nicht-Engagierten ( $n=16.173$ ).

Zwischen den verschiedenen Gruppen mit Migrationshintergrund zeigen sich zudem weitere Unterschiede. Überdurchschnittlich hoch ist die Engagementbereitschaft bei Personen, die keine deutsche Staatsangehörigkeit haben, und zwar sowohl in der Gruppe mit eigener Zuwanderungserfahrung als auch in der Gruppe ohne eigene Zuwanderungserfahrung. Am höchsten sind die Anteile Engagementbereiter bei Per- sonen ohne deutsche Staatsangehörigkeit mit eigener Zuwanderungserfahrung. Von diesen sind 60,0 Prozent vielleicht und 15,3 Prozent sicher bereit sich zukünftig zu engagieren. Diese hohe Bereitschaft, sich später einmal im Aufnahmeland Deutschland zu engagieren, kann auch als Bereitschaft zur gesellschaftlichen Teilhabe und Integration verstanden werden (Abbildung 23-4). 


\subsection{Ausgestaltung des freiwilligen Engagements nach Migrationshintergrund}

Welche Unterschiede zeigen sich zwischen Engagierten mit Migrationshintergrund und Engagierten ohne Migrationshintergrund? Engagieren sich Menschen mit Migrationshintergrund in anderen gesellschaftlichen Bereichen als Engagierte ohne Migrationshintergrund, und wenn ja, in welchen? Haben Engagierte mit Migrationshintergrund gleich häufig eine Leitungsfunktion wie Engagierte ohne Migrationshintergrund? Wenden sie jeweils gleich viel Zeit für ihr Engagement auf? Diesen Fragen gehen wir im Folgenden nach.

\section{Abbildung 23-5: Anteile der Engagierten in vierzehn gesellschaftlichen Bereichen 2014, nach Migrationshintergrund}

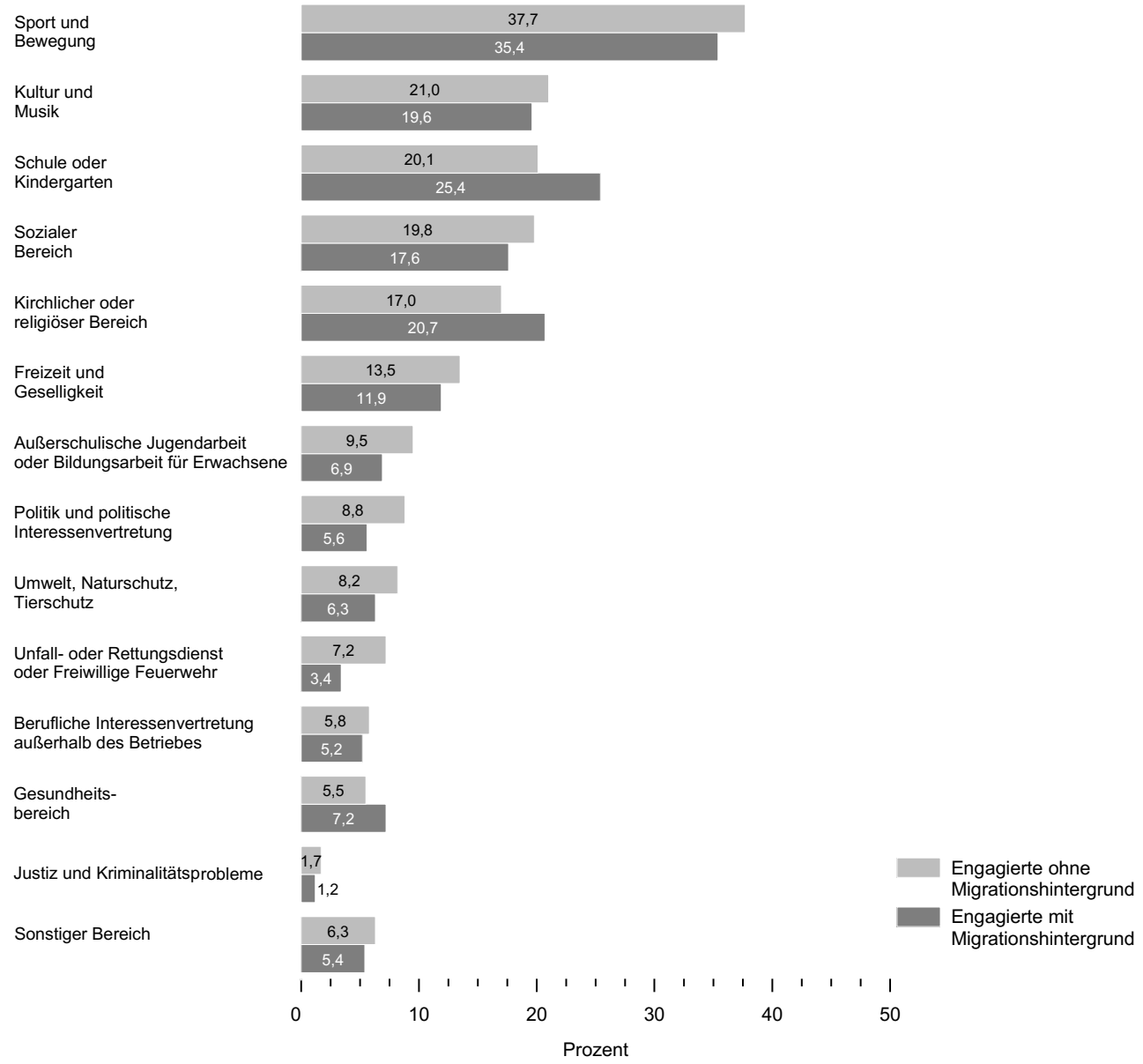

Quelle: FWS 2014, gewichtet, eigene Berechnungen (DZA). Basis: Alle Engagierten $(n=12.516)$. 


\subsubsection{Engagementbereiche}

Engagierte mit Migrationshintergrund betätigen sich überwiegend in denselben gesellschaftlichen Bereichen wie Engagierte ohne Migrationshintergrund. Es zeigen sich viele Gemeinsamkeiten und nur wenige Unterschiede zwischen diesen beiden Engagiertengruppen. Beide Gruppen sind zum Beispiel am häufigsten im Bereich Sport und Bewegung freiwillig oder ehrenamtlich tätig (Abbildung 23-5). Darüber hinaus zeigen sich graduelle Unterschiede, etwa in den Bereichen Schule und Kindergarten sowie im kirchlichen oder religiösen Bereich. Engagierte mit Migrationshintergrund übernehmen in diesen beiden Bereichen anteilig häufiger Aufgaben als Engagierte ohne Migrationshintergrund. $\mathrm{Zu}$ deutlich geringeren Anteilen als Engagierte ohne Migrationshintergrund sind Engagierte mit Migrationshintergrund insbesondere im Bereich der Unfall- und Rettungsdienste sowie der Freiwilligen Feuerwehr tätig, hier scheint noch Nachholbedarf seitens der kommunalen Akteure zu bestehen, auch Menschen mit Migrationshintergrund für diese Aufgaben zu gewinnen.

Bezüglich der Art des Migrationshintergrundes zeigen sich keine einheitlichen Muster in den verschiedenen Engagementbereichen (Abbildung 23-6). Im Bereich Sport und Bewegung sind zum Beispiel ausländische Engagierte mit Zuwanderungserfahrung zu gleichen Teilen engagiert wie deutsche Engagierte mit Migrationshintergrund, aber ohne eigene Zuwanderungserfahrung. Hier zeigt sich die besondere Integrationsleistung der Sportinfrastruktur in Deutschland. 


\section{Abbildung 23-6: Anteile der Engagierten in vierzehn gesellschaftlichen Bereichen 2014, nach Art des Migrationshintergrundes}

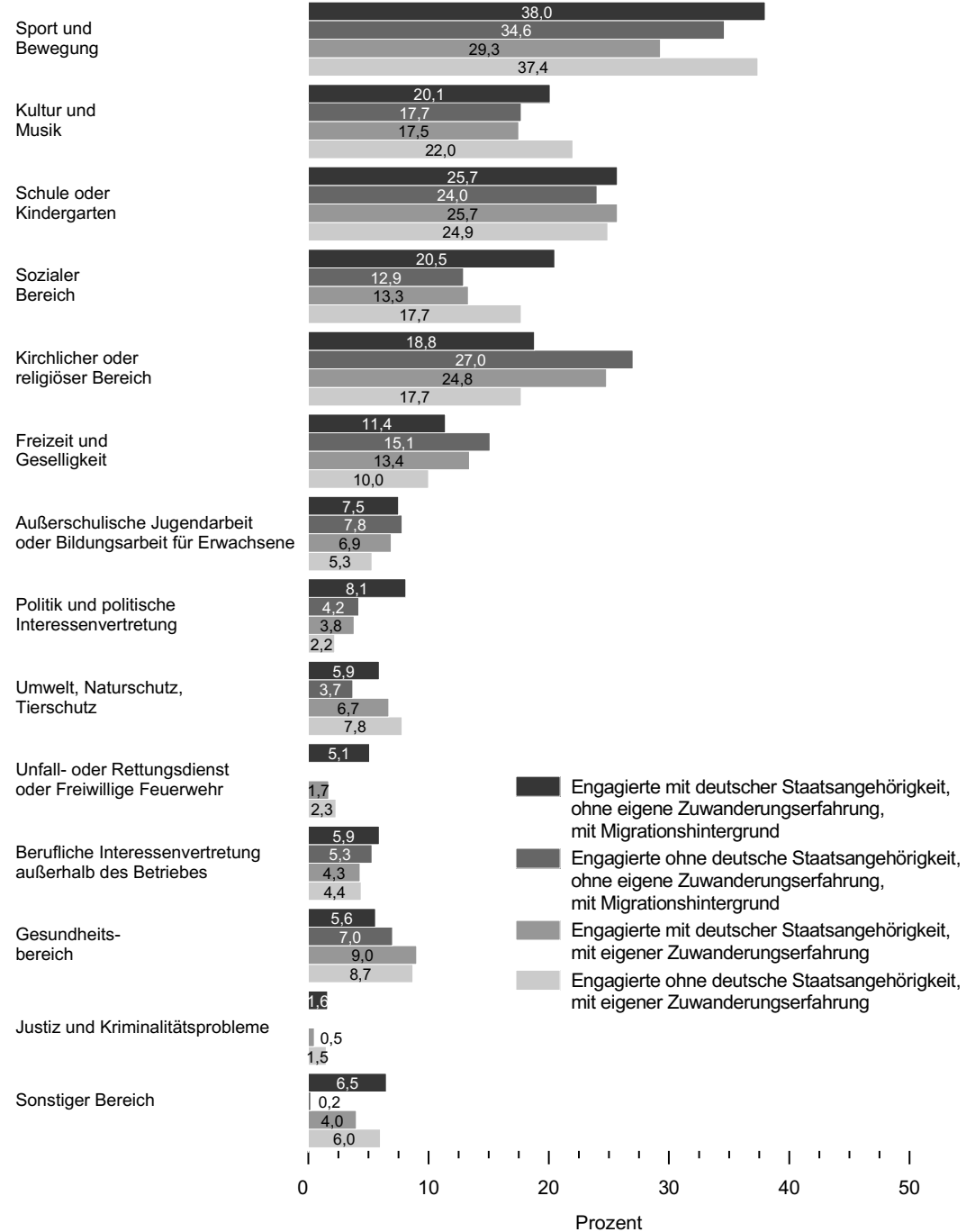

Quelle: FWS 2014, gewichtet, eigene Berechnungen (DZA). Basis: Alle Engagierten mit Migrationshintergrund $(n=1.616)$. 


\subsubsection{Leitungsfunktion im freiwilligen Engagement}

Obwohl sich Engagierte mit Migrationshintergrund in ähnlichen Bereichen betätigen wie Engagierte ohne Migrationshintergrund, haben sie anteilig seltener eine Leitungsfunktion im freiwilligen Engagement (Abbildung 23-7). Dabei haben sowohl Frauen (19,4 Prozent) als auch Männer (23,1 Prozent) mit Migrationshintergrund anteilig seltener eine Leitungsfunktion im Engagement als Frauen (22,3 Prozent) beziehungsweise Männer (34,7 Prozent) ohne Migrationshintergrund. Der Geschlechterunterschied hinsichtlich der Leitungsfunktionen ist aber bei Personen mit Migrationshintergrund mit 3,7 Prozentpunkten wesentlich schwächer ausgeprägt als bei Personen ohne Migrationshintergrund (Differenz von 12,4 Prozentpunkten; siehe Tabelle 23-6a im Online-Anhang). Besonders gering ist der Anteil Engagierter mit Leitungs- oder Vor- standsfunktion bei den in Deutschland geborenen Personen, die keine deutsche Staatsangehörigkeit haben. Obwohl davon auszugehen ist, dass der Anteil bei dieser Gruppe noch steigen wird, weil sie sich durch ein besonders geringes Durchschnittsalter auszeichnet und der Anteil der Engagierten mit Leitungs- oder Vorstandsfunktion im mittleren Lebensalter höher ist (siehe Kapitel 11), überrascht dieser Unterschied: Schließlich haben diese Engagierten ohne eigene Zuwanderungserfahrung in der Regel in Deutschland die Schule besucht und die zivilgesellschaftliche Infrastruktur wie Vereine und Einrichtungen kennengelernt. Allerdings sind nicht nur Diskriminierungsmechanismen als Erklärung für diese Gruppenunterschiede zu debattieren, sondern es können sich zum Beispiel auch Bildungs- und Berufsunterschiede in der Übernahme von Leitungs- und Vorstandsfunktionen im Engagement niederschlagen.

\section{Abbildung 23-7: Anteile Engagierter mit Leitungs- oder Vorstandsfunktion nach Art des Migrationshintergrundes 2014}

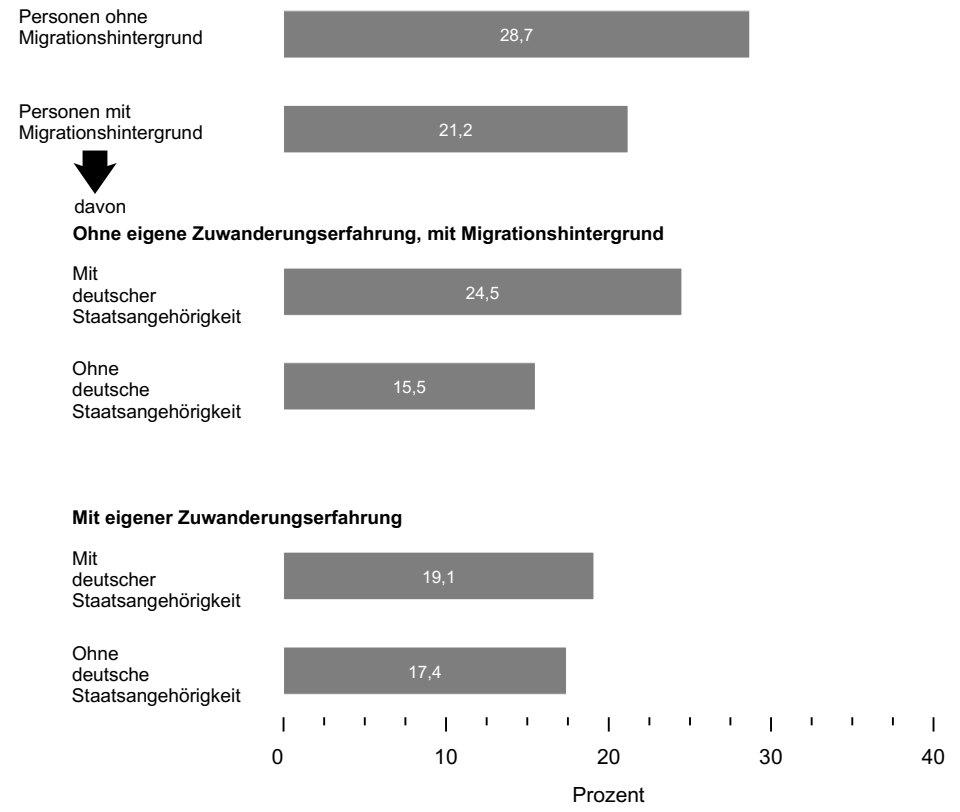

Quelle: FWS 2014, gewichtet, eigene Berechnungen (DZA). Basis: Alle Engagierten $(n=12.516)$. 


\subsubsection{Zeitaufwand}

Engagierte mit Migrationshintergrund sind nicht nur in ähnlichen gesellschaftlichen Bereichen wie Engagierte ohne Migrationshintergrund freiwillig tätig, sondern sie wenden auch in etwa gleich viel Zeit für ihre freiwillige Tätigkeit auf: Weit mehr als die Hälfte der Engagierten widmet der freiwilligen Tätigkeit wöchentlich bis zu zwei Stunden (Abbildung 23-8). Der höchste Zeitaufwand findet sich bei den deutschen Engagierten mit Migrationshintergrund, aber ohne eigene Zuwanderungserfahrung: Mehr als jede beziehungsweise jeder Fünfte von ihnen engagiert sich in einem zeitlichen Umfang von sechs und mehr Stunden pro Woche. Insgesamt sind Menschen mit Migrationshintergrund zwar zu geringeren Anteilen freiwillig engagiert als Menschen ohne Migrationshintergrund, aber das Engagement derjenigen, die sich engagieren, unterscheidet sich in Bezug auf den Stundenumfang kaum.

\section{Abbildung 23-8: Zeitaufwand für die freiwillige Tätigkeit nach Art des Migrationshintergrundes 2014}

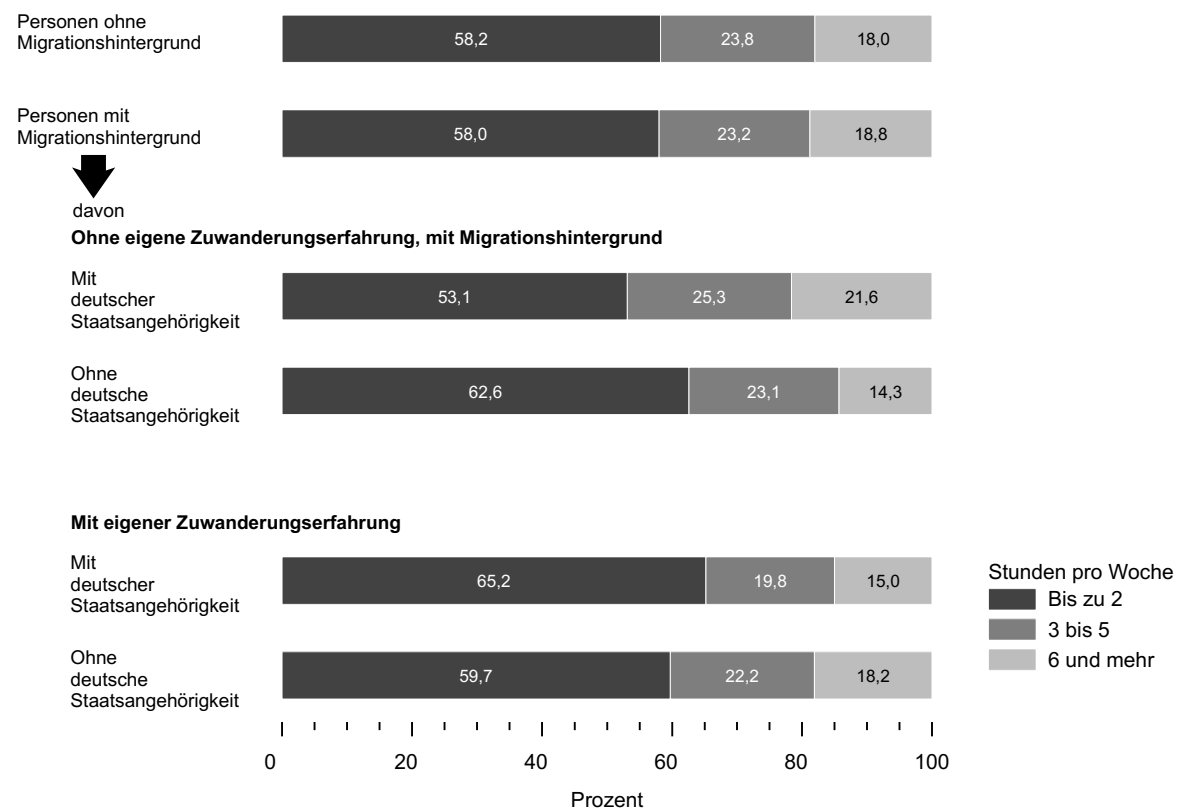

Quelle: FWS 2014, gewichtet, eigene Berechnungen (DZA). Basis: Alle Engagierten $(n=12.516)$. 


\subsection{Informelle Unterstützung für Nachbarinnen und Nachbarn, Freundinnen und Freunde, Bekannte und Andere nach Migrationshintergrund}

\subsubsection{Instrumentelle Hilfen}

Instrumentelle Hilfen für Nachbarinnen und Nachbarn, Freundinnen und Freunde, Bekannte und Andere werden von 23,0 Prozent der Menschen mit Migrationshintergrund und von 27,0 Prozent der Menschen ohne Migrationshintergrund erbracht (Abbildung 23-9). Während sich der Anteil in der Gruppe der Deutschen mit Migrationshintergrund, aber ohne eigene $\mathrm{Zu}$ wanderungserfahrung (27,4 Prozent) kaum von dem Anteil in der Gruppe der Deutschen ohne Migrationshintergrund unterscheidet (27,0 Pro- zent), sind die Anteile in den anderen Gruppen mit Migrationshintergrund deutlich kleiner (Abbildung 23-9). Von den Personen ohne deutsche Staatsangehörigkeit und ohne eigene Zuwanderungserfahrung haben 24,1 Prozent in den vergangenen zwölf Monaten Nachbarinnen und Nachbarn, Freundinnen und Freunden oder Bekannten geholfen. Von den Personen mit eigener Zuwanderungserfahrung leisten solche Hilfen 20,4 Prozent der Personen ohne deutsche Staatsangehörigkeit und 20,1 Prozent der Deutschen mit eigener Zuwanderungserfahrung.

Abbildung 23-9: Anteile der Personen, die instrumentelle Hilfen für Nachbarinnen und Nachbarn, Freundinnen und Freunde oder Bekannte leisten, 2014, nach Art des Migrationshintergrundes

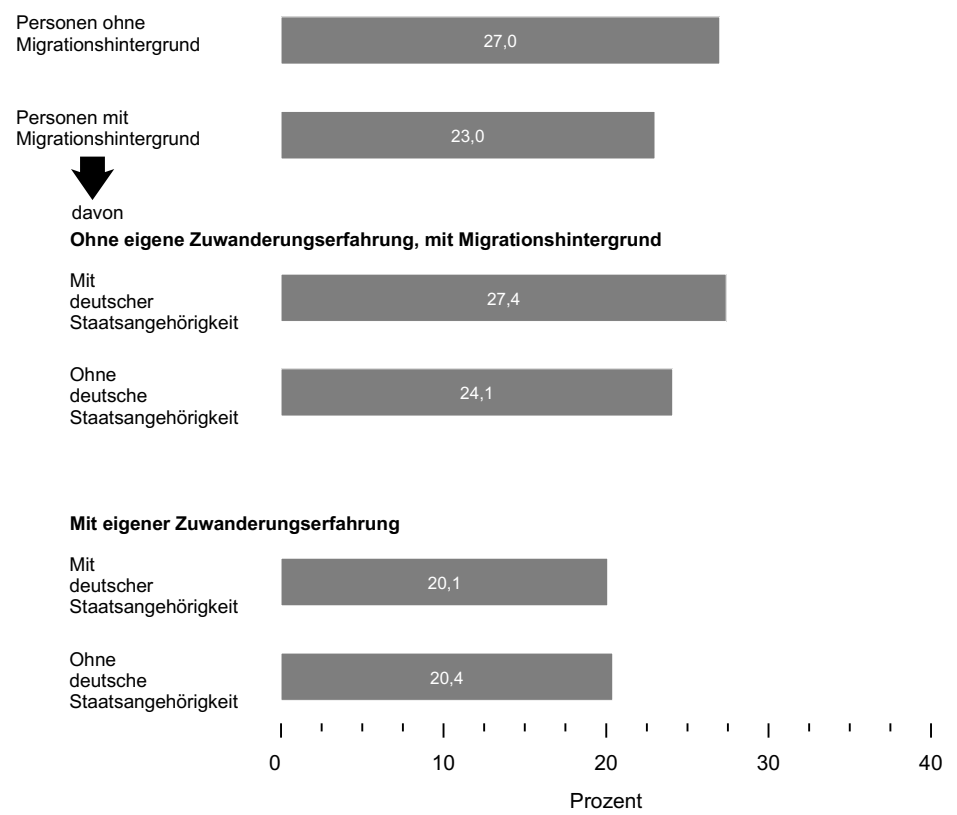

Quelle: FWS 2014, gewichtet, eigene Berechnungen (DZA). Basis: Alle Befragten ( $n=28.690)$. 


\subsubsection{Kinderbetreuung für nicht-eigene Kinder}

Insgesamt betreuen 21,1 Prozent der in Deutschland lebenden Personen mit Migrationshintergrund Kinder, die nicht ihre eigenen sind, zum Beispiel Kinder aus der Nachbarschaft oder dem Freundeskreis (Abbildung 23-10). Kinder von Verwandten sind hier ebenso wie die eigenen Kinder ausgenommen. In der Gruppe der Deutschen ohne Migrationshintergrund beträgt dieser Anteil 18,5 Prozent, Personen mit Migrationshintergrund betreuen also anteilig etwas häufiger nicht-eigene Kinder.

\section{Abbildung 23-10: Anteile der Personen, die Kinderbetreuung für Nachbarinnen und Nachbarn, Freundinnen und Freunde oder Bekannte leisten, 2014, nach Art des Migrationshintergrundes}

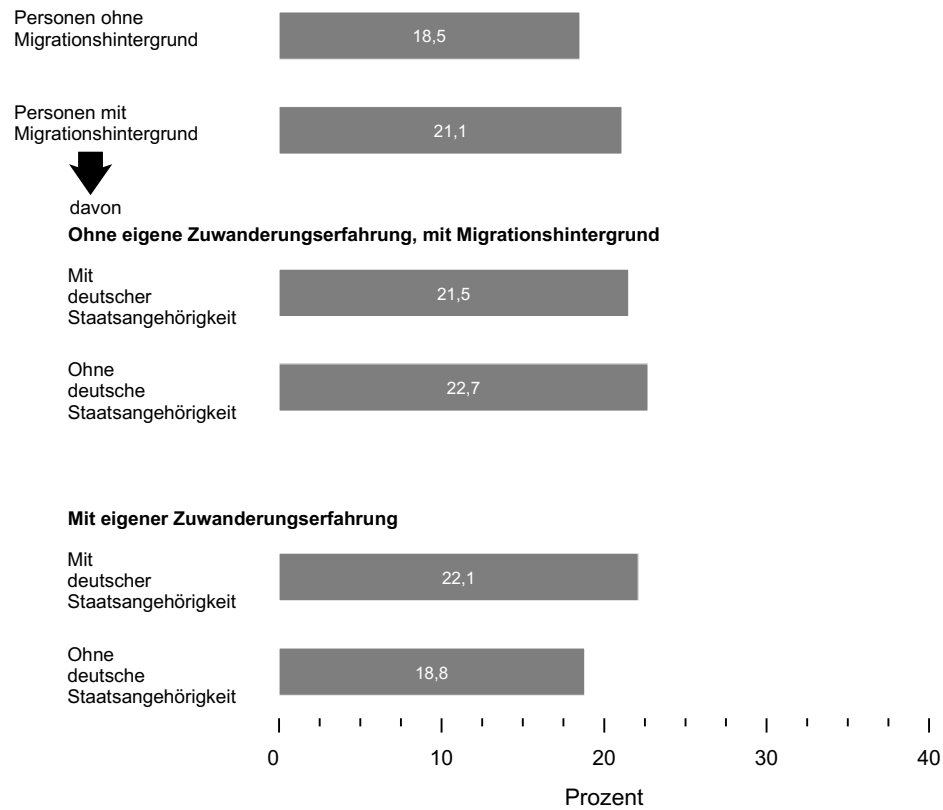

Quelle: FWS 2014, gewichtet, eigene Berechnungen (DZA). Basis: Alle Befragten ( $n=28.690)$.

Bezüglich der Kinderbetreuung zeigen sich auch Unterschiede zwischen Gruppen mit unterschiedlichem Migrationshintergrund, diese sind jedoch relativ gering: In der Gruppe der Ausländerinnen und Ausländer ohne eigene Zuwanderungserfahrung liegt der Anteil mit 22,7 Prozent am höchsten (Abbildung 23-10). Dieses Ergebnis ist insbesondere vor dem Hintergrund plausibel, als dass die Personen ohne eigene Zuwanderungserfahrung im Schnitt jünger sind als Personen mit eigener Zuwanderungserfahrung, und die Betreuung von nicht-eigenen Kindern meist von Personen übernommen wird, die selbst auch eigene minderjährige Kinder haben. Bei den Personen ohne deutsche Staatsangehörigkeit und mit eigener Zuwanderungserfahrung betreuen lediglich 18,8 Prozent Kinder von Nachbarinnen und Nachbarn, Freundinnen und Freunden, Bekannten und Anderen.

In den Gruppen ohne deutsche Staatsangehörigkeit ist die Differenz zwischen den Betreuungsanteilen der Frauen und Männer zudem größer als in den Gruppen mit deutscher Staatsangehörigkeit (Tabelle 23-7b im Online-Anhang). Zum Beispiel betreuen 19,2 Prozent der Männer ohne deutsche Staatsangehörigkeit und 
ohne eigene Zuwanderungserfahrung Kinder im sozialen Nahraum, aber 26,6 Prozent der Frauen mit dem gleichen Migrationshintergrund; ein Geschlechterunterschied von 7,4 Prozentpunkten. In der Gruppe der Personen ohne deutsche Staatsangehörigkeit mit eigener Zuwanderungserfahrung beträgt der Unterschied sogar 9,1 Prozentpunkte. In dieser Gruppe betreuen 23,4 Prozent der Frauen und 14,3 Prozent der Männer Kinder von Nachbarinnen und Nachbarn, Freundinnen und Freunden, Bekannten oder Anderen. Die geschlechtsspezifischen Unterschiede im Betreuungsverhalten sind bei deutschen Staatsangehörigen weniger stark ausgeprägt als bei anderen Staatsangehörigen, sie haben jedoch dieselbe Richtung und folgen somit ebenfalls traditionellen Mustern der geschlechtsspezifischen Arbeitsteilung.

Am häufigsten werden Kinder im sozialen Nahraum von Personen betreut, die selbst in einem jüngeren oder mittleren Lebensalter sind (siehe Kapitel 9): In der Altersgruppe der 30- bis 49-Jährigen variieren die Anteile bei Personen mit Migrationshintergrund zwischen geringen 18,7 Prozent bei Ausländerinnen und Ausländern ohne eigene Zuwanderungserfahrung und hohen 28,9 Prozent bei Deutschen mit eigener Zuwanderungserfahrung. In der Altersgruppe der 14- bis 29-Jährigen liegen die Anteile jeweils etwas darunter, mit einer Ausnahme, nämlich der Gruppe der Ausländerinnen und Ausländer ohne eigene Zuwanderungserfahrung, hier beträgt der Anteil 28,1 Prozent und liegt somit über dem in der Altersgruppe der 30-bis 49-Jährigen (Tabelle 23-7b im Online-Anhang).

Am seltensten werden Kinder für Nachbarinnen und Nachbarn, Freundinnen und Freunde oder Bekannte in allen Migrationsgruppen durch Menschen im Alter von 65 und mehr Jahren betreut, die Anteile variieren lediglich zwischen 6,9
Prozent bei den Personen ohne deutsche Staatsangehörigkeit mit eigener Zuwanderungserfahrung und 8,1 Prozent bei den Deutschen mit eigener Zuwanderungserfahrung (Tabelle 23-7b im Online-Anhang).

\subsubsection{Pflege und Betreuung}

Die Übernahme von Pflege- und Betreuungstätigkeiten für Nachbarinnen und Nachbarn, Freundinnen und Freunde, Bekannte oder Andere ist ein vergleichsweise seltenes Phänomen. Lediglich 3,2 Prozent der Personen mit Migrationshintergrund und 3,5 Prozent der Personen ohne Migrationshintergrund übernehmen solche Tätigkeiten (Abbildung 23-11). In allen betrachteten Gruppen pflegen oder betreuen Frauen anteilig häufiger Personen im sozialen Nahraum als Männer: 4,1 Prozent der Frauen versus 2,3 Prozent der Männer mit Migrationshintergrund im Vergleich zu 4,3 Prozent der Frauen versus 2,6 Prozent der Männer ohne Migrationshintergrund (Tabelle 23-8a im Online-Anhang). In beiden Bevölkerungsteilen wird die Pflege am häufigsten von Personen im Alter von 65 Jahren und älter übernommen (Tabelle 23-8a im Online-Anhang).

Im Vergleich der vier verschiedenen Gruppen mit Migrationshintergrund variiert der Anteil der Pflegenden zwischen lediglich 0,7 Prozent bei Personen ohne deutsche Staatsangehörigkeit und ohne eigene Zuwanderungserfahrung und dem höchsten Anteil von jeweils 3,6 Prozent bei Personen ohne deutsche Staatsangehörigkeit mit eigener Zuwanderungserfahrung sowie bei Deutschen mit Migrationshintergrund, aber ohne eigene Zuwanderungserfahrung (Abbildung 23-11). 


\section{Abbildung 23-11: Anteile der Personen, die Pflege oder Betreuung für Nachbarinnen und Nachbarn, Freundinnen und Freunde oder Bekannte leisten, 2014, nach Art des Migrationshintergrundes}

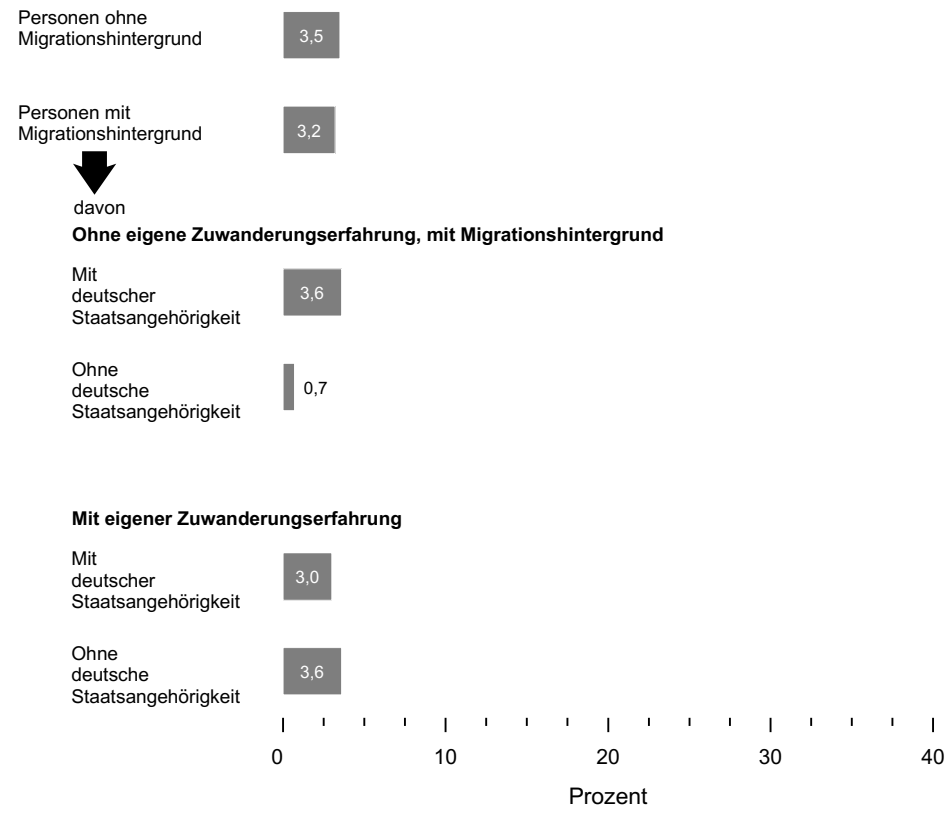

Quelle: FWS 2014, gewichtet, eigene Berechnungen (DZA). Basis: Alle Befragten ( $n=28.690)$.

Die Unterschiede zwischen Menschen mit Migrationshintergrund und Menschen ohne Migrationshintergrund fallen bezüglich der informellen Unterstützungsleistungen vergleichsweise gering aus. Die Unterschiede bezüglich des freiwilligen Engagements und der zivilgesellschaftlichen Aktivität sind viel größer als die Unterschiede bei Unterstützungsleistungen im sozialen Nahraum. Zwar leisten Personen mit Migrationshintergrund anteilig geringfügig seltener praktische Hilfen für Nachbarinnen und Nachbarn, Freundinnen und Freunde, Bekannte oder Andere, aber bei Pflege- und Betreuungsleistungen zeigt sich kein Unterschied und die Kinderbetreuung übernehmen sie sogar zu etwas höheren Anteilen. Bei gemeinsamer Betrachtung aller Unterstützungsarten leisten Personen mit Migrationshintergrund also zu sehr ähnlichen Anteilen Unterstützung wie Personen ohne Migrationshintergrund.
Die zu beobachtenden Unterschiede hinsichtlich der informellen Unterstützungsleistungen unter den Menschen mit Migrationshintergrund scheinen in erster Linie Unterschiede in der Geschlechter- und Altersgruppenzusammensetzung der vier Gruppen mit Migrationshintergrund widerzuspiegeln: Personen mit eigener Zuwanderungserfahrung sind im Schnitt älter als Personen mit Migrationshintergrund ohne eigene Zuwanderungserfahrung. Darüber hinaus zeigen sich in unterschiedlicher Schärfe in allen Gruppen dieselben geschlechtsspezifischen Muster: Frauen betreuen und pflegen anteilig häufiger, Männer helfen anteilig häufiger bei praktischen Arbeiten außerhalb des Haushaltes (siehe Kapitel 9). Hinweise auf eine Kompensation des geringeren Anteils freiwilligen Engagements durch einen höheren Anteil an informeller Unterstützung im sozialen Nahraum zeigen sich allerdings nicht. 


\subsection{Multivariate Analyse des freiwilligen Engagements in Abhängigkeit vom Migrationshintergrund}

Bislang haben wir deskriptive Vergleiche vorgenommen. Im Mittelpunkt standen dabei vier Gruppen von Personen mit Migrationshintergrund. Diese vier Gruppen unterscheiden sich aber nicht allein mit Blick auf Migrationserfahrung und Staatsbürgerschaft, sondern auch hinsichtlich weiterer Merkmale. Geschlecht, Alter und Bildung sind in diesen vier Gruppen unterschiedlich verteilt. Darüber hinaus unterscheiden sich die Gruppen nach weiteren Aspekten der Lebenssituation, beispielsweise nach der Einkommenslage, der gesundheitlichen Situation oder der Zusammensetzung ihres Freundesund Bekanntenkreises (Baykara-Krumme 2012; Fuhr 2012; Rommel, Saß \& Born 2015). Aus diesem Grund werden die Gruppenvergleiche durch multivariate Analysen ergänzt, in denen für Geschlecht, Alter und Bildung sowie weitere Merkmale kontrolliert wird.

In Tabelle 23-3 ist ein logistisches Regressionsmodell zur Vorhersage des freiwilligen Engagements ( $\mathrm{ja} / \mathrm{nein})$ dargestellt. In dieses Modell gehen als unabhängige Variablen der Migrationshintergrund ein, sowie die Variablen Geschlecht, Alter und Schulbildung. Als Referenzgruppe für die Testung der Effekte wurde die Gruppe der Männer im Alter von 50 bis 64 Jahren mit mittlerem Bildungsniveau gewählt. Es wurde dabei diejenige Gruppe als Bezugspunkt gewählt, die im Alter und in der Bildung in einer mittleren Position liegt (daher wurde die Gruppe der Personen im mittleren Alter mit mittlerer Bildung gewählt). Zudem wurde die Gruppe der Männer als Referenzgröße gewählt, um die Ergebnisse für die Gruppe der Frauen explizit darstellen zu können (wird die Referenzgruppe Frauen gewählt, kann kein Koeffizient für Frauen ausgewiesen werden). Zudem bezieht dieses Mo- dell als Kontrollvariablen Merkmale mit ein, die sich ebenfalls als relevant für die Wahrscheinlichkeit, sich freiwillig zu engagieren, erwiesen haben (subjektive Einkommenssituation, subjektive Gesundheit, Netzwerkqualität, solidarische Werthaltung; siehe Kapitel 16 bis 19) sowie die Design-Variablen Bundesland und Kontakt über Festnetz/Mobilfunk, die für die Stichprobenziehung des Freiwilligensurveys von Bedeutung sind, aber an dieser Stelle nicht inhaltlich interpretiert werden (siehe Kapitel 2).

In der Ergebnistabelle sind die im Modell geschätzten unstandardisierten Koeffizienten dargestellt. Positive Koeffizienten (zum Beispiel ,mit Migrationshintergrund, ohne Zuwanderungserfahrung und mit deutscher Staatsangehörigkeit': 0,85) zeigen an, dass eine positive Beziehung zwischen dem Merkmal und freiwilligem Engagement vorliegt: Bei in Deutschland geborenen Personen mit Migrationshintergrund mit deutscher Staatsangehörigkeit, ist die Wahrscheinlichkeit, freiwillig engagiert zu sein, höher als in der Referenzgruppe (Menschen mit Migrationshintergrund, mit Zuwanderungserfahrung, ohne deutsche Staatsangehörigkeit). Negative Vorzeichen der Koeffizienten zeigen an, dass eine negative Beziehung des Merkmals zum Engagement vorliegt, Personen also eine im Vergleich zur Referenzgruppe geringere Wahrscheinlichkeit haben, sich freiwillig zu engagieren. Je größer der Betrag eines Koeffizienten ist, desto stärker ist der untersuchte Zusammenhang. Dargestellt sind nur jene Koeffizienten, bei denen ein Signifikanztest angezeigt hat, dass der betreffende Koeffizient statistisch bedeutsam ist (nicht signifikante Koeffizienten werden durch drei Striche angezeigt). 
Tabelle 23-3: Ergebnisse logistischer Regression, Vorhersage freiwilligen Engagements durch Migrationshintergrund, Geschlecht, Alter und Bildung 2014

\begin{tabular}{lc}
\hline $\begin{array}{l}\text { Migrationshintergrund (Referenz: Mit Migrationshintergrund, } \\
\text { mit Zuwanderungserfahrung, ohne deutsche Staatsangehörigkeit) }\end{array}$ & $1,11^{* * *}$ \\
\hline Ohne Migrationshintergrund & $0,85^{* * *}$ \\
\hline $\begin{array}{l}\text { Mit Migrationshintergrund, ohne Zuwanderungserfahrung } \\
\text { und mit deutscher Staatsangehörigkeit }\end{array}$ & --- \\
\hline $\begin{array}{l}\text { Mit Migrationshintergrund, ohne Zuwanderungserfahrung } \\
\text { und ohne deutsche Staatsangehörigkeit }\end{array}$ & --- \\
\hline $\begin{array}{l}\text { Mit Migrationshintergrund, mit Zuwanderungserfahrung } \\
\text { und mit deutscher Staatsangehörigkeit }\end{array}$ & \\
\hline Geschlecht (Referenz: Männer) & $-0,24^{* * *}$ \\
\hline Frauen & $-0,09^{*}$ \\
\hline Alter (Referenz: 50 bis 64 Jahre) & $0,13^{* * *}$ \\
\hline 14 bis 29 Jahre & $-0,28^{* * *}$ \\
\hline 30 bis 49 Jahre & \\
\hline 65 Jahre und älter & $0,56^{* * *}$ \\
\hline Bildung (Referenz: Mittlere Bildung) & $-0,42^{* * *}$ \\
\hline Noch Schüler/in & $0,44^{* * *}$ \\
\hline Niedrige Bildung & 0,07 \\
\hline Hohe Bildung & 27.565 \\
\hline Pseudo R2 & \\
\hline Anzahl gültiger Fälle & \\
\hline
\end{tabular}

Quelle: FWS 2014, ungewichtet, eigene Berechnung (DZA). Kontrolliert für subjektives Einkommen, Netzwerkqualität, Werthaltung Solidarität, subjektive Gesundheit sowie Design-Variablen (Bundesland, Kontakt über Festnetz/Mobilfunk). ${ }^{*} p<0,05 ; * * p<0,01 ; * * *<0,001 ;$--- nicht signifikant.

In den Ergebnissen zeigt sich, dass die gefundenen Unterschiede zwischen den Migrationsgruppen weitgehend stabil bleiben, auch wenn weitere Aspekte der Lebenssituation einbezogen werden. Wenn die Unterschiede zwischen den verschiedenen Gruppen mit Migrationshintergrund etwa ausschließlich durch Bildungsunterschiede verursacht wären, würden der Zusammenhang zwischen Migrationshintergrund und freiwilligem Engagement verschwinden, oder sich zumindest deutlich abschwächen, wenn zusätzlich Bildung im Modell kontrolliert wird. Gegenüber einem Grundmodell, das zusätzlich zum Migrationshintergrund nur die Design-Variablen Bundesland und Kontakt über Festnetz/Mobilfunk berücksichtigt (Modell nicht dargestellt), ändern sich die Koeffizienten aber nur minimal bei Kontrolle von Alter, Geschlecht und Bildung. Auch unter Berücksichtigung der zusätzlichen Kontrollvariablen sind Deutsche ohne Migrationshintergrund also mit höherer Wahrscheinlichkeit freiwillig engagiert als die hier herangezogene Referenzgruppe (Personen mit Migrationshintergrund, die im Ausland geboren sind und 
keine deutsche Staatsangehörigkeit haben). Wir haben diese Gruppe als Referenz gewählt, weil sie in der deskriptiven Betrachtung den geringsten Anteil freiwillig Engagierter aufweist (vgl. Abbildung 23-3). Außerdem ist die Gruppe von Personen mit Migrationshintergrund, ohne Zuwanderungserfahrung, aber mit deutscher Staatsangehörigkeit mit höherer Wahrscheinlichkeit freiwillig engagiert als die Referenzgruppe. Offensichtlich sind prägende Erfahrungen in Kindheit und Jugend, die Personen mit und ohne

\subsection{Fazit}

\subsubsection{Engagement von Menschen mit Migrationshintergrund: Erfolgreiche Integration in der zweiten Generation?}

Deutschland ist ein Einwanderungsland mit einem hohen Anteil von Menschen mit Migrationshintergrund. Diese Gruppe ist aber keineswegs homogen: Wir unterscheiden zwischen Menschen mit einem Migrationshintergrund der ersten und der zweiten Generation. Personen aus der ersten Migrationsgeneration haben selbst die Migrationserfahrung gemacht, Personen aus der zweiten Generation sind in Deutschland geboren und aufgewachsen, und zwar in einer Familie, in der die Eltern die Migrationserfahrung gemacht haben. Zudem ist berücksichtigt, ob Personen mit Migrationshintergrund die deutsche Staatsbürgerschaft haben oder nicht.

Diskutiert man die Beteiligung am freiwilligen Engagement als Grad- und Erfolgsmesser der Integration, so lässt sich sagen: Integration braucht Zeit - und zwar bis in die zweite Generation. Zugleich ist festzuhalten, dass Integration auch einen sicheren, anerkannten Status benötigt: die deutsche Staatsbürgerschaft. Integrationserfolge zeigen sich anhand der Engagementquote, die am höchsten ist bei Menschen mit eigene Migrationserfahrung gemacht haben, von erheblicher Bedeutung für das freiwillige Engagement. Ebenfalls wichtig ist die Bedeutung der deutschen Staatsangehörigkeit in Kombination mit dem Geburtsland Deutschland, denn die Gruppe derjenigen mit Migrationshintergrund, ohne Zuwanderungserfahrung, aber auch ohne deutsche Staatsangehörigkeit unterscheidet sich unter Kontrolle aller Einflussfaktoren nicht von der Referenzgruppe (mit Zuwanderungserfahrung, ohne deutsche Staatsangehörigkeit).

Migrationshintergrund in der zweiten Generation, die zugleich die deutsche Staatsangehörigkeit haben. Wer in Deutschland sozialisiert wurde, hat einen besseren Zugang zu zivilgesellschaftlichen Organisationen und Tätigkeitsfeldern als Personen, die erst als Erwachsene nach Deutschland zugewandert sind. Trotz durchschnittlich langer Aufenthaltsdauer in Deutschland ist die erste Generation der Zuwanderinnen und $\mathrm{Zu}$ wanderer im Engagement nicht so stark vertreten wie die zweite Generation. Auch die Staatsangehörigkeit macht einen Unterschied, jüngere Ausländerinnen und Ausländer ohne eigene $\mathrm{Zu}$ wanderungserfahrung könnten möglicherweise besser im Engagement integriert werden, wenn sie auch die deutsche Staatsangehörigkeit hätten oder diese Zugangsbarriere im freiwilligen Engagement anderweitig abgebaut werden könnte. Möglicherweise sind auch in dieser Gruppe der in Deutschland Geborenen und Aufgewachsenen ohne deutsche Staatsangehörigkeit die Gelegenheiten zum freiwilligen Engagement nicht so bekannt wie bei gleichaltrigen Personen mit deutscher Staatsangehörigkeit. Gleichzeitig soll an dieser Stelle darauf verwiesen werden, dass freiwilliges Engagement offensichtlich nicht als alleiniger Gradmesser für Integration betrachtet werden kann, denn auch Personen, die sich nicht 
freiwillig engagieren, können bestens integriert sein, und zwar unabhängig davon, ob sie einen Migrationshintergrund haben oder nicht.

Die Gründe, warum Personen mit Migrationshintergrund anteilig seltener freiwillig engagiert sind als Personen ohne Migrationshintergrund, sind jedoch nicht monokausal, sondern vielfältig und auf verschiedenen Ebenen angesiedelt. Die geringere Beteiligung wird in der Literatur auf einen Mix von bestehenden Barrieren zurückgeführt (Gensicke 2015; Gluns, Pinkert, Wilmes \& Rass 2012; Habermann, Brink-Mann, Schäfer \& Stagge 2013; Halm \& Sauer 2007; Huth 2011, 2012a, 2012b; May \& Alisch 2013). Häufig wird auf Unterschiede in der individuellen Ressourcenausstattung der Personen verwiesen - wer etwa von Einsamkeit und Armutsgefährdung, von Arbeitslosigkeit oder Analphabetismus betroffen ist, geringe deutsche Sprachkenntnisse oder einen ungeklärten Aufenthaltsstatus in Deutschland hat, wird sich mit hoher Wahrscheinlichkeit nicht freiwillig engagieren; ohne deutsche Staatsangehörigkeit gilt in manchen Bereichen sogar, dass sich Personen nicht engagieren dürfen.

Diskutiert wird auch, welche kulturellen Unterschiede es hinsichtlich der Frage gibt, ob das Übernehmen einer freiwilligen Aufgabe überhaupt als freiwilliges Engagement wahrgenommen und bezeichnet wird. Möglicherweise lernen Personen, die das Prinzip des zivilgesellschaftlichen Engagements aus ihrem Herkunftsland nicht kennen, erst im Gespräch darüber oder im öffentlichen Diskurs in Deutschland, dass ein vertrautes Alltagshandeln (etwa das Übernehmen einer freiwilligen Aufgabe in der Kirche oder der Moschee oder die Hilfe für sozial Schwache) hier als freiwilliges Engagement verstanden werden kann. Sicher ist, dass sich die gebräuchlichen Bezeichnungen für freiwilliges Engagement in verschiedenen Sprachen stark unterscheiden. Diese Faktoren könnten weitere Erklärungsbausteine dafür sein, dass bestimmte Personen mit Migrationshintergrund in Studien wie dem Freiwilligensurvey seltener ein Engagement angeben als Personen ohne Migrationshin- tergrund. Der Erhebungseffekt sollte aber insofern eher gering sein, da die zweistufige Abfrage der Aktivität und des Engagements nach Mitmachen in gesellschaftlichen Bereichen mit entsprechenden Beispielen und nach der Übernahme von Arbeiten und Aufgaben sehr umfangreich gestellt ist und Begriffe wie freiwilliges oder ehrenamtliches Engagement im Telefoninterview vermieden werden (siehe Kapitel 2).

Darüber hinaus wird auf Defizite der zivilgesellschaftlichen Organisationen verwiesen, die es in der Vergangenheit teils versäumt haben oder bei denen teils keine Bereitschaft besteht, sich interkulturell zu öffnen (Ilgün-Birhimeoglu 2014). Ausnahmen etwa im Bereich der Sportvereine zeigen, dass die oft konstatierten Nachwuchsprobleme der Vereine und Verbände durch das gezielte Anwerben von Personen mit Migrationshintergrund gelöst werden können. Dies ist vor allem deswegen ein sehr vielversprechender Ansatz, da die Bereitschaft, sich zu engagieren, bei Menschen mit Migrationshintergrund vergleichsweise groß ist und somit einen idealen Anknüpfungspunkt für Integrationsmaßnahmen darstellt. Die hohe Engagementbereitschaft verweist aber auch auf Zugangsbarrieren, die noch bestehen. Da Menschen mit Migrationshintergrund auch $\mathrm{zu}$ geringeren Anteilen öffentlich aktiv sind, und dies als Voraussetzung für Engagement betrachtet werden kann, sollten sich Organisationen sowohl bezüglich der Engagierten aber auch bezüglich der Menschen, die einfach erstmal nur mitmachen wollen, interkulturell öffnen. Zudem sollte die Kooperation zwischen Organisationen verbessert werden, so dass auch Migrantenorganisationen in lokale Netwerke einbezogen werden (Huth 2013). Dass sich Menschen mit Migrationshintergrund anteilig seltener freiwillig engagieren, scheint also zumindest nicht daran zu liegen, dass ihr Interesse am Engagement geringer ist, denn die Engagementbereitschaft ist bei den Nicht-Engagierten mit Migrationshintergrund sogar höher als bei den Nicht-Engagierten ohne Migrationshintergrund. Darüber hinaus gibt es eine große Zahl von Migrantenorganisationen, in denen sich 
Menschen mit Migrationshintergrund freiwillig engagieren (Koopmans, Dunkel, Schaeffer \& Veit 2011).

Hinsichtlich der Ausgestaltung des freiwilligen Engagements zeigen sich wesentlich mehr Gemeinsamkeiten von Engagierten mit und ohne Migrationshintergrund als Unterschiede. Menschen mit Migrationshintergrund engagieren sich in den großen gesellschaftlichen Bereichen (z. B. Sport und Bewegung sowie Schule und Kindergarten) ebenso am häufigsten wie Menschen ohne Migrationshintergrund. Zudem setzen Engagierte auch einen ähnlichen Stundenumfang für ihre Tätigkeit ein. Unterschiede zeigen sich jedoch bezüglich der Leitungs- oder Vorstandsfunktion, eine solche haben Engagierte mit Migrationshintergrund anteilig seltener. Hier besteht ein großes Potenzial für zivilgesellschaftliche Organisationen, die insbesondere Personen für die Übernahme solcher freiwilliger Tätigkeiten mit Leitungsaufgaben suchen.

\subsubsection{Implikationen}

Da Engagierte mit Migrationshintergrund in manchen Engagementbereichen deutlich unterrepräsentiert sind, ist es notwendig, dort bestehende Zugangsbarrieren zu analysieren und abzubauen. Eine wichtige Bedingung hierfür ist, dass anders als in der Vergangenheit, in der Personen mit Migrationshintergrund in einer Defizitperspektive häufig allein als Empfängerinnen und Empfänger von Hilfen betrachtet wurden, der Blick darauf gerichtet wird: Auch Menschen mit Migrationshintergrund sind Unterstützungsgeberinnen und -geber, und zwar unabhängig von ihrer Herkunft und ihrer Staatsangehörigkeit.

Eine weitere wichtige Bedingung ist, gegebene Zugangsbarrieren zu benennen. Ein Beispiel: Für den Bereich der Freiwilligen Feuerwehr stellt Klausing (2010) dar, dass unter den Personen mit Migrationshintergrund ein Informationsdefizit bezüglich der Möglichkeiten besteht, sich in der Jugendfeuerwehr und der Freiwilligen Feuerwehr betätigen zu können. Entweder sind die
Möglichkeiten, sich bei der Feuerwehr freiwillig $\mathrm{zu}$ engagieren, überhaupt nicht bekannt, oder die Feuerwehr wird als traditionelle deutsche Einrichtung wahrgenommen, vielleicht auch als ethnisch homogene, von der sich Menschen mit Migrationshintergrund nicht angesprochen beziehungsweise nicht einbezogen fühlen. Bislang fehlen hier meist die entsprechenden zielgruppenspezifischen Zugangswege. Dem könnten die kommunalen Akteure durch entsprechende Informationsangebote Abhilfe schaffen, die auf Jugendliche und deren Interessen zugeschnitten sein müssen und die Engagementinteressierten mit Migrationshintergrund auch in ihrem sozialen Umfeld erreichen müssen, um so Personen aus dieser Bevölkerungsgruppe zu gewinnen. Selbst für Deutsche mit Migrationshintergrund ist es eben nicht selbstverständlich, dass ihnen die Angebote und Möglichkeiten der zivilgesellschaftlichen Landschaft in Deutschland vertraut sind. Eine weitere viel diskutierte Bedingung ist, dass die zivilgesellschaftlichen Organisationen sich interkulturell öffnen müssen, um auch für Menschen mit Migrationshintergrund dauerhaft attraktiv zu sein, dies erfordert in der Regel von allen Beteiligten, sich aufeinander einzulassen. Darauf, dass die Abschaffung oder die Überbrückung von Zugangsbarrieren wirkungsvoll sein wird, verweist in jedem Fall auch die hohe Engagementbereitschaft bei Menschen mit Migrationshintergrund.

Zugangsbarrieren können aber auch dergestalt vorhanden sein, dass Menschen mit Migrationshintergrund im Durchschnitt über geringere sozioökonomische (beispielsweise finanzielle) Ressourcen verfügen als Personen ohne Migrationshintergrund und deshalb nicht die gleichen Möglichkeiten haben, sich freiwillig zu engagieren. Eine stärkere Öffnung zivilgesellschaftlicher Organisationen für Personengruppen mit geringeren Ressourcen käme daher auch vielen Menschen mit Migrationshintergrund zugute.

Abschließend möchten wir eine Aussage wiederholen, die wir bereits am Anfang des Kapitels gemacht haben: Die Gruppen der Personen mit Migrationshintergrund sind sehr unterschied- 
lich. Im vorliegenden Kapitel wurden Personen mit Migrationshintergrund nach ihrer Zuwanderungserfahrung und ihrer Staatsangehörigkeit differenziert. Heterogenität zeigt sich - zum Teil abgeleitet daraus, zum Teil aber auch unabhängig davon - aber auch in vielen anderen Dimensionen, beispielsweise in Fragen des Sprachgebrauchs, der Kultur oder der sozioökonomischen
Lage. Diese Vielfalt kann einerseits eine Herausforderung für die Förderung des freiwilligen Engagements von Menschen mit Migrationshintergrund darstellen. Sie kann andererseits und im gelingenden Fall der Integration von Migrantinnen und Migranten in zivilgesellschaftliche Strukturen aber auch eine Bereicherung für das freiwillige Engagement und die Gesellschaft sein.

\section{Literatur}

Baykara-Krumme, H. (2012). Die Bedeutung der Migrationserfahrung für die soziale Einbindung im Alter. In: H. Baykara-Krumme, A. Motel-Klingebiel \& P. Schimany (Hrsg.) Viele Welten des Alterns. Ältere Migranten im alternden Deutschland (S. 255-287). Wiesbaden: Springer VS.

Baykara-Krumme, H. (2010). Interviewereffekte in Bevölkerungsumfragen. Ein Beitrag zur Erklärung des Teilnahme- und Antwortverhaltens von Migranten (Arbeitspapier des Beziehungs- und Familienpanels). Chemnitz: pairfam. Panel Analysis of Intimate Relationships and Family Dynamics.

Bundesamt für Migration und Flüchtlinge (BAMF) (2015). Konzept für einen bundesweiten Alphabetisierungskurs. Nürnberg: BAMF.

Bundesamt für Migration und Flüchtlinge (BAMF) (2016). Migrationsbericht des Bundesamtes für Migration und Flüchtlinge im Auftrag der Bundesregierung (Migrationsbericht 2014). Nürnberg: BAMF.

Bundesamt für Migration und Flüchtlinge (BAMF) (2010). Engagiert für Integration. Erkenntnisse und Handlungsempfehlungen aus 16 Modellprojekten zum interkulturellen bürgerschaftlichen Engagement. Nürnberg: BAMF.

Freitag, M., Manatschal, A., Ackermann, K., \& Ackermann, M. (2016). Freiwilligenmonitor Schweiz 2016. Zürich: Seismo.

Fuhr, G. (2012). Armutsgefährdung von Menschen mit Migrationshintergrund. Wirtschaft und Statistik, Juli 2012, 549-563.

Gensicke, T. (2015). Freiwilliges Engagement in Deutschland: Freiwilligensurvey 2009. Wiesbaden: Springer VS.

Gluns, D., Pinkert, C., Wilmes, M., \& Rass, C. (2012). MigrantInnen als Zielgruppe ehrenamtlichen Engagements im Zivilund Katastrophenschutz. Studie im Auftrag des Bundesamtes für Bevölkerungsschutz und Katastrophenhilfe. Osnabrück: Institut für Migrationsforschung und Interkulturelle Studien (IMIS) Universität Osnabrück.

Habermann, M., Brink-Mann, D., Schäfer, G., \& Stagge, M. (2013). Integration, Prävention und Partizipation - niedrigschwellige Angebotsentwicklung für Migrant/innen im Stadtteil: Ein Werkstattbericht. Sozialmagazin. Themenheft Älter werden - älter sein. (5/6), 38-47.

Halm, D., \& Sauer, M. (2007). Bürgerschaftliches Engagement von Türkinnen und Türken in Deutschland. Wiesbaden: VS Verlag für Sozialwissenschaften.

Haug, S. (2013). Migration. In: S. Mau \& N. M. Schöneck (Hrsg.) Handwörterbuch zur Gesellschaft Deutschlands (S. 593607). Wiesbaden: Springer VS.

Huth, S. (2013). Vergleichende Fallstudien zum freiwilligen Engagement von Menschen mit Migrationshintergrund (eNewsletter Wegweiser Bürgergesellschaft 9/2013). Online: http://www.buergergesellschaft.de/fileadmin/pdf/ gastbeitrag_huth_130524.pdf (zuletzt abgerufen 24.2.2016).

Huth, S. (2012a). Bürgerschaftliches Engagement von älteren MigrantInnen. In: Heinrich-Böll-Stiftung (Hrsg.) DOSSIER Altern in der Migrationsgesellschaft (S. 27-31). Berlin: Heinrich-Böll-Stiftung.

Huth, S. (2012b). Freiwilliges und bürgerschaftliches Engagement von Menschen mit Migrationshintergrund - Barrieren und Türöffner. Bonn: Friedrich-Ebert-Stiftung.

Huth, S. (2011). Migration und Integration. In: T. Olk \& B. Hartnuß (Hrsg.) Handbuch Bürgerschaftliches Engagement (S. 439-449). Weinheim: Beltz Juventa. 
Ilgün-Birhimeoglu, E. (2014). Interkulturelle Öffnung in Organisationen des ehrenamtlichen Engagements. In: E. Vanderheiden \& C.-H. Mayer (Hrsg.) Handbuch interkulturelle Öffnung (S. 373-382). Göttingen: Vandenhoeck \& Ruprecht.

Klausing, J. (2010). Einstellungs- und Interessenslagen von Jugendlichen mit Migrationshintergrund zum ehrenamtlichen Engagement in der Jugendfeuerwehr in Niedersachsen. Hannover: Niedersächsisches Ministerium für Inneres, Sport und Integration.

Koopmans, R., Dunkel, A., Schaeffer, M., \& Veit, S. (2011). Ethnische Diversität, soziales Vertrauen und Zivilengagement (Discussion Paper SP IV 2011-703). Berlin: Wissenschaftszentrum Berlin für Sozialforschung.

Lämmermann, F. (2013). Einbürgerung. In: K.-H. Meier-Braun \& R. Weber (Hrsg.) Migration und Integration in Deutschland (S. 117-119). Bonn: Bundeszentrale für politische Bildung.

May, M., \& Alisch, M. (2013). AMIQUS - unter Freunden. Ältere Migrantinnen und Migranten in der Stadt. Opladen: Barbara Budrich.

Rommel, A., Saß, A. C., Born, S., \& Ellert, U. (2015). Die gesundheitliche Lage von Menschen mit Migrationshintergrund und die Bedeutung des sozioökonomischen Status. Bundesgesundheitsblatt Gesundheitsforschung, Gesundheitsschutz, 58(6), 543-552.

Simonson, J., Hagen, C., Vogel, C., \& Motel-Klingebiel, A. (2013). Ungleichheit sozialer Teilhabe im Alter. Zeitschrift für Gerontologie und Geriatrie, 46(5), 410-416.

Statistisches Bundesamt (2015). Bevölkerung und Erwerbstätigkeit. Bevölkerung mit Migrationshintergrund. Ergebnisse des Mikrozensus. Wiesbaden: Statistisches Bundesamt.

Tucci, I. (2013). Lebenssituation von Migranten und deren Nachkommen. In: Statistisches Bundesamt \& Wissenschaftszentrum Berlin für Sozialforschung (Hrsg.) Datenreport 2013. Ein Sozialbericht für die Bundesrepublik Deutschland (S. 198-204). Bonn: Bundeszentrale für politische Bildung.

United Nations Educational, Scientific and Cultural Organization (UNESCO) (2015). Bildung für Alle 2000-2015: Bilanz. Bonn: Deutsche UNESCO-Kommission.

Vogel, C. (2012). Generationenbeziehungen der (Spät-)Aussiedler - Forschungsstand und exemplarische Befund zu Einstellungen in Bezug auf familiale Unterstützungsleistungen Befunde. In: H. Baykara-Krumme, A. Motel-Klingebiel \& P. Schimany (Hrsg.) Viele Welten des Alterns. Ältere Migranten im alternden Deutschland (S. 289-313). Wiesbaden: Springer VS.

Open Access Dieses Kapitel wird unter der Creative Commons Namensnennung 4.0 International Lizenz (http://creativecommons.org/licenses/by/4.0/deed.de) veröffentlicht, welche die Nutzung, Vervielfältigung, Bearbeitung, Verbreitung und Wiedergabe in jeglichem Medium und Format erlaubt, sofern Sie den/die ursprünglichen Autor(en) und die Quelle ordnungsgemäß nennen, einen Link zur Creative Commons Lizenz beifügen und angeben, ob Änderungen vorgenommen wurden.

Die in diesem Kapitel enthaltenen Bilder und sonstiges Drittmaterial unterliegen ebenfalls der genannten Creative Commons Lizenz, sofern sich aus der Abbildungslegende nichts anderes ergibt. Sofern das betreffende Material nicht unter der genannten Creative Commons Lizenz steht und die betreffende Handlung nicht nach gesetzlichen Vorschriften erlaubt ist, ist für die oben aufgeführten Weiterverwendungen des Materials die Einwilligung des jeweiligen Rechteinhabers einzuholen. 